TITLE:

\title{
Slow evaporation and condensation on a spherical droplet in the presence of a noncondensable gas
}

$\operatorname{AUTHOR}(\mathrm{S})$ :

Kosuge, Shingo; Aoki, Kazuo; Hatano, Masatake

\section{CITATION:}

Kosuge, Shingo ... [et al]. Slow evaporation and condensation on a spherical droplet in the presence of a noncondensable gas. Physics of Fluids 2010, 22(6): 067101.

\section{ISSUE DATE:}

2010

URL:

http://hdl.handle.net/2433/126660

RIGHT:

(C) 2010 American Institute of Physics 


\title{
Slow evaporation and condensation on a spherical droplet in the presence of a noncondensable gas
}

\author{
Shingo Kosuge, ${ }^{1, a)}$ Kazuo Aoki, ${ }^{1}$ and Masatake Hatano ${ }^{2}$ \\ ${ }^{1}$ Department of Mechanical Engineering and Science \\ and Advanced Research Institute of Fluid Science and Engineering, Graduate School of Engineering, \\ Kyoto University, Kyoto 606-8501, Japan \\ ${ }^{2}$ Department of Aeronautics and Astronautics, Graduate School of Engineering, \\ Kyoto University, Kyoto 606-8501, Japan
}

(Received 6 October 2009; accepted 16 February 2010; published online 14 June 2010)

\begin{abstract}
A spherical droplet is placed in a binary mixture composed of the vapor of the droplet and another gas which neither evaporates nor condenses (a noncondensable gas). The mixture is in an equilibrium state at rest at infinity. A slow steady flow of the vapor caused by weak evaporation or condensation, under the influence of the noncondensable gas, is investigated on the basis of a linearized model Boltzmann equation. Numerical analyses by means of a finite-difference method are carried out for a wide range of the Knudsen number (i.e., from a large to small droplet compared to the molecular mean free path). The numerical results, together with analytical solutions for small and large Knudsen numbers, clarify the behavior the mixture, i.e., the mass- and heat-flow rates from or onto the droplet as well as spatial distributions of the macroscopic quantities, in the entire range of gas rarefaction. The solution for the steady heat transfer problem between a solid sphere and a binary gas mixture is also obtained as a byproduct. (C) 2010 American Institute of Physics.
\end{abstract}

[doi:10.1063/1.3432130]

\section{INTRODUCTION}

A vapor flow around its condensed phase is one of the major subjects of research in molecular gas dynamics (or rarefied gas dynamics). Since the state of the vapor at an interface where net evaporation or condensation is taking place may deviate significantly from an equilibrium, one cannot apply conventional gas dynamics (i.e., the NavierStokes set of equations) and should employ molecular gas dynamics based on the Boltzmann equation. As described in Refs. 1-6, fundamental problems for evaporating or condensing vapor flows, such as the flow in a half space in contact with a plane condensed phase (see, e.g., Refs. 7-15 and Sec. 6.1 in Ref. 6), that between two parallel plane condensed phases (e.g., Refs. 16-18), and that around a cylindrical or spherical condensed phase (see, e.g., Refs. 19-28 and Secs. 6.2-6.4 in Ref. 6), have extensively been studied in the framework of molecular gas dynamics during past decades. Those problems were first considered in the case of a single-component system (i.e., a pure vapor and its condensed phase). Then part of the analysis has been extended to the case of mixtures, i.e., the case of a binary mixture of vapor and noncondensable gas $^{29-32}$ or a mixture of vapors, ${ }^{33-35}$ because of the practical importance as well as of the theoretical interest for the mixtures.

In the present paper, we will focus on vapor flows evaporating from or condensing onto its spherical condensed phase (a droplet) in the presence of a noncondensable gas. There is a long history of studies on this problem tracing back to Maxwell because of its fundamental importance in connection with the droplet growth or evaporation phenom-

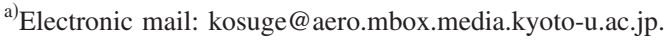

enon appearing in a wide area of science and technology. The detailed analysis of this problem may contribute directly or indirectly toward the better understanding of physics of, e.g., atmospheric aerosols ${ }^{36,37}$ and fuel droplets in internal combustion engines $^{38,39}$ and toward a precise modeling of compressible flows with phase transition. ${ }^{40}$ However, the theoretical studies so far have been based mostly on a simple physical model (e.g., the Langmuir model employed in Refs. 41 and 42 or the similar type of model in Ref. 39) or on rough analyses of the Boltzmann equation using the Maxwell moment method ${ }^{19,43,44}$ or the Grad moment method. ${ }^{45,46}$ In addition, many of the preceding works based on the Boltzmann equation (or its model equation) have been concerned only with the limited case where concentration of the vapor is very low, ${ }^{47-49}$ or the case where the Knudsen number (the molecular mean free path divided by droplet's radius) is very small. ${ }^{50,51}$ Reliable solutions of the Boltzmann equation for a wide range of parameters (i.e., for intermediate Knudsen numbers, arbitrary concentration of the vapor, and so on) have not been reported yet.

In the present study, under the assumption that evaporation and condensation are weak, we carry out a direct numerical analysis of the linearized model Boltzmann equation by means of a finite-difference method. The discontinuity of the velocity distribution function of molecules, which is a phenomenon generally observed around a convex body, ${ }^{25,52}$ is accurately handled in the computation by use of the hybrid scheme devised in Ref. 25. The numerical solutions for intermediate Knudsen numbers thus obtained and the theoretical solutions for large and small Knudsen numbers (Ref. 50) together clarify the behavior of the vapor and noncondensable gas around the droplet in the whole range of the Knudsen number. Specifically, the mass and heat fluxes from the 
droplet are obtained as functions of the Knudsen number and are compared with Young's approximate formula proposed in Ref. 42. The profiles of the macroscopic quantities, such as the density, pressure, temperature, etc., are also obtained.

This paper is organized as follows. The physical setting of the problem and assumptions are described and are formulated in Sec. II. The analyses are carried out in Sec. III. Some relations resulting from Onsager's reciprocity relation and the principle of increase in entropy are introduced in Sec. III A, solutions for the free-molecular gas and for small Knudsen numbers are introduced in Sec. III B, and key issues in the numerical analysis for intermediate Knudsen numbers are summarized briefly in Sec. III C. The results of the analyses and the data of numerical computations are given in Sec. IV. Finally, a generalization of the boundary condition on the surface of the droplet is discussed in Sec. V and the paper is concluded in Sec. VI.

\section{FORMULATION}

\section{A. Problem and assumptions}

Consider a spherical droplet with radius $L$ and temperature $T_{w}$, placed in a binary mixture composed of the vapor of the droplet (gas $A$ ) and another noncondensable gas (gas $B$ ). The mixture is in an equilibrium state at rest with temperature $T_{0}$, partial pressure of the vapor $p_{0}^{A}$, and that of the noncondensable gas $p_{0}^{B}$ at infinity. We investigate the steady flow of the vapor caused by evaporation or condensation on the droplet and the behavior of the noncondensable gas under the following assumptions: (i) the behavior of the mixture is described by the model Boltzmann equation proposed by Hamel $^{53}$ or that proposed by Garzó et al. ${ }^{54}$ (ii) the vapor molecules leaving the surface of the droplet are distributed according to the stationary Maxwellian distribution with temperature $T_{w}$ and pressure $p_{w}^{A}$, where $p_{w}^{A}$ is the saturated vapor pressure of gas $A$ at temperature $T_{w}$ (the complete condensation condition); (iii) the noncondensable-gas molecules are reflected diffusely on the surface of the droplet; and (iv) $\left|p_{w}^{A}-p_{0}^{A}\right| / p_{0}^{A}$ and $\left|T_{w}-T_{0}\right| / T_{0}$ are small, so that the equation and the boundary condition can be linearized around the equilibrium state at infinity.

Note that the model equation of Hamel and that of Garzó et al. become identical once the linearization around an equilibrium state at rest is made. This means that, no matter which of the two models we choose, the basic equation for the present problem [under assumption (iv)] will be given by the same equation, i.e., Eq. (2) appearing in Sec. II C. It should be also noted that we will not use the relation between the saturated vapor pressure $p_{w}^{A}$ and temperature $T_{w}$ of the droplet (i.e., the Clausius-Clapeyron relation) and treat them as if they were independent parameters in the following analyses.

\section{B. Notation}

Let us summarize the main notation used in the paper. In the sequel, the Greek letters $\alpha$ and $\beta$ will be used symbolically to represent the gas species, i.e., $\{\alpha, \beta\}=\{A, B\}$. $n_{0}^{\alpha}$ is the molecular number density of gas $\alpha$ at infinity and is given by $n_{0}^{\alpha}=p_{0}^{\alpha} / \kappa T_{0}$, where $\kappa$ is the Boltzmann constant. $(\operatorname{Lr}, \theta, \varphi)$ is the spherical coordinate system with its origin at the center of the droplet, $m^{\alpha}$ is the molecular mass of gas $\alpha$, and $\left(2 \kappa T_{0} / m^{A}\right)^{1 / 2} \zeta$ is the molecular-velocity vector. The magnitude and components of $\zeta$ are denoted by $\zeta(=|\zeta|)$ and $\left(\zeta_{r}, \zeta_{\theta}, \zeta_{\varphi}\right)$, respectively. $f_{0}^{\alpha}\left(1+\Phi^{\alpha}\right)$ is the velocity distribution function of $\alpha$-gas molecules, where $f_{0}^{\alpha}$ is the reference distribution (i.e., the equilibrium state at infinity) expressed as $f_{0}^{\alpha}=n_{0}^{\alpha}\left(m^{A} / 2 \kappa T_{0}\right)^{3 / 2} E^{\alpha}$ with $E^{\alpha}=\left(\hat{m}^{\alpha} / \pi\right)^{3 / 2} \exp \left(-\hat{m}^{\alpha} \zeta^{2}\right)$ and $\hat{m}^{\alpha}=m^{\alpha} / m^{A}$. Note that $\left|\Phi^{\alpha}\right| \ll 1$ under assumption (iv). In the case of the Hamel or Garzó et al. model equation, the collision frequency of an $\alpha$-gas molecule for collisions with $\beta$-gas molecules in the reference state is given by $K^{\alpha \beta} n_{0}^{\beta}$ with $K^{\alpha \beta}\left(=K^{\beta \alpha}\right)$ being a positive constant. Then, we define the reference mean free path $\ell_{0}^{A}$ of the vapor molecules as $\ell_{0}^{A}=\left(8 \kappa T_{0} / \pi m^{A}\right)^{1 / 2} /\left(K^{A A} n_{0}^{A}+K^{A B} n_{0}^{B}\right)$. With $n_{0}^{\alpha}, K^{\alpha \beta}$, and $\ell_{0}^{A}$, we define $\hat{n}_{0}^{\alpha}=n_{0}^{\alpha} / n_{0}^{A}, \hat{K}^{\alpha \beta}=K^{\alpha \beta} / K^{A A}$, and $k=(\sqrt{\pi} / 2) \mathrm{Kn}$ $=(\sqrt{\pi} / 2)\left(\ell_{0}^{A} / L\right)$, where $\mathrm{Kn}$ is the Knudsen number. The molecular number density, flow velocity, temperature, pressure, stress tensor, and heat-flow vector of gas $\alpha$ are denoted by $n_{0}^{\alpha}\left(1+\omega^{\alpha}\right), \quad\left(2 \kappa T_{0} / m^{A}\right)^{1 / 2} u_{i}^{\alpha}, \quad T_{0}\left(1+\tau^{\alpha}\right), \quad p_{0}^{\alpha}\left(1+P^{\alpha}\right), \quad p_{0}^{\alpha}\left(\delta_{i j}\right.$ $\left.+P_{i j}^{\alpha}\right)$, and $p_{0}^{\alpha}\left(2 \kappa T_{0} / m^{A}\right)^{1 / 2} Q_{i}^{\alpha}$, respectively, where $\{i, j\}$ $=\{r, \theta, \varphi\}$ and $\delta_{i j}$ is the Kronecker delta. The corresponding quantities of the mixture are similarly denoted by $n_{0}(1+\omega)$, $\left(2 \kappa T_{0} / m^{A}\right)^{1 / 2} u_{i}, \quad T_{0}(1+\tau), \quad p_{0}(1+P), \quad p_{0}\left(\delta_{i j}+P_{i j}\right), \quad$ and $p_{0}\left(2 \kappa T_{0} / m^{A}\right)^{1 / 2} Q_{i}$, where $n_{0}=n_{0}^{A}+n_{0}^{B}$ and $p_{0}=p_{0}^{A}+p_{0}^{B}$.

\section{Basic equation and boundary condition}

Because of the spherical symmetry of the problem, one may expect that $\Phi^{\alpha}$ depends only on $r, \zeta_{r}$, and $\left(\zeta_{\theta}^{2}+\zeta_{\varphi}^{2}\right)^{1 / 2}$. Indeed, the solution $\Phi^{\alpha}$ can be sought in the following form (similarity solution):

$$
\Phi^{\alpha}=\Phi^{\alpha}\left(r, \zeta, \theta_{\zeta}\right),
$$

where $\theta_{\zeta}\left(0 \leq \theta_{\zeta} \leq \pi\right)$ is the angle between the vector $\zeta$ and $r$-direction, namely, $\theta_{\zeta}=\cos ^{-1}\left(\zeta_{r} / \zeta\right)$. The compatibility of form (1) with the problem can be checked by direct substitutions into the linearized Boltzmann equation (or its model equation) and the boundary conditions (see Refs. 28 and 55).

By use of the notation introduced in Sec. II B and the similarity solution (1), the linearized version of the Hamel or Garzó et al. model equation for the present problem may be written as follows (see the second paragraph in Sec. II A): for $\alpha=A, B$,

$$
\begin{aligned}
\zeta \cos \theta_{\zeta} \frac{\partial \Phi^{\alpha}}{\partial r}-\frac{\zeta \sin \theta_{\zeta}}{r} \frac{\partial \Phi^{\alpha}}{\partial \theta_{\zeta}} \\
=\frac{1}{\left(1+\hat{K}^{A B} \hat{n}_{0}^{B}\right) k} \sum_{\beta=A, B} \hat{K}^{\alpha \beta} \hat{n}_{0}^{\beta}\left(\Psi^{\alpha \beta}-\Phi^{\alpha}\right),
\end{aligned}
$$

where

$$
\begin{aligned}
& \Psi^{\alpha \beta}=\omega^{\alpha}+2 \hat{m}^{\alpha} \zeta \cos \theta_{\zeta} u_{r}^{\alpha \beta}+\left(\hat{m}^{\alpha} \zeta^{2}-\frac{3}{2}\right) \tau^{\alpha \beta}, \\
& u_{r}^{\alpha \beta}=\frac{\hat{m}^{\alpha} u_{r}^{\alpha}+\hat{m}^{\beta} u_{r}^{\beta}}{\hat{m}^{\alpha}+\hat{m}^{\beta}},
\end{aligned}
$$




$$
\tau^{\alpha \beta}=\tau^{\alpha}+\frac{2 \hat{m}^{\alpha} \hat{m}^{\beta}}{\left(\hat{m}^{\alpha}+\hat{m}^{\beta}\right)^{2}}\left(\tau^{\beta}-\tau^{\alpha}\right) .
$$

The expressions of $\omega^{\alpha}, u_{r}^{\alpha}$, and $\tau^{\alpha}$ in terms of $\Phi^{\alpha}$, which complete Eq. (2), are given in Eq. (8) appearing later.

The boundary condition on the surface of the droplet is written as follows: at $r=1$, for $0 \leq \theta_{\zeta}<\pi / 2$,

$$
\begin{aligned}
& \Phi^{A}=\Delta P+\left(\zeta^{2}-\frac{5}{2}\right) \Delta T, \\
& \Phi^{B}=\left(\hat{m}^{B} \zeta^{2}-2\right) \Delta T-2\left(\pi \hat{m}^{B}\right)^{1 / 2}\left\langle\zeta \cos \theta_{\zeta} \Phi^{B} E^{B}\right\rangle_{w},
\end{aligned}
$$

where

$$
\Delta P=\left(p_{w}^{A}-p_{0}^{A}\right) / p_{0}^{A}, \quad \Delta T=\left(T_{w}-T_{0}\right) / T_{0},
$$

and $\langle\cdots\rangle_{w}$ is defined for a function $\phi\left(\zeta, \theta_{\zeta}\right)$ as

$$
\langle\phi\rangle_{w} \equiv 2 \pi \int_{0}^{\infty} \int_{\pi / 2}^{\pi} \phi \zeta^{2} \sin \theta_{\zeta} \mathrm{d} \theta_{\zeta} \mathrm{d} \zeta
$$

Note that $|\Delta P|$ and $|\Delta T|$ are small [i.e., assumption (iv)]. On the other hand, the condition at infinity is given by

$$
\Phi^{\alpha} \rightarrow 0 \quad \text { as } r \rightarrow \infty .
$$

The macroscopic quantities of gas $\alpha$ are expressed in terms of moments of $\Phi^{\alpha}$ as

$$
\begin{aligned}
& \omega^{\alpha}=\left\langle\Phi^{\alpha} E^{\alpha}\right\rangle, \quad u_{r}^{\alpha}=\left\langle\zeta \cos \theta_{\zeta} \Phi^{\alpha} E^{\alpha}\right\rangle, \\
& \tau^{\alpha}=\frac{2}{3}\left\langle\left(\hat{m}^{\alpha} \zeta^{2}-\frac{3}{2}\right) \Phi^{\alpha} E^{\alpha}\right\rangle, \\
& P_{r r}^{\alpha}=2 \hat{m}^{\alpha}\left\langle\zeta^{2} \cos ^{2} \theta_{\zeta} \Phi^{\alpha} E^{\alpha}\right\rangle, \\
& P_{\theta \theta}^{\alpha}=P_{\varphi \varphi}^{\alpha}=\frac{3 P^{\alpha}-P_{r r}^{\alpha}}{2}, \quad P^{\alpha}=\omega^{\alpha}+\tau^{\alpha}, \\
& Q_{r}^{\alpha}=\left\langle\zeta \cos \theta_{\zeta}\left(\hat{m}^{\alpha} \zeta^{2}-\frac{5}{2}\right) \Phi^{\alpha} E^{\alpha}\right\rangle,
\end{aligned}
$$

with $\langle\cdots\rangle$ being defined as

$$
\langle\phi\rangle \equiv 2 \pi \int_{0}^{\infty} \int_{0}^{\pi} \phi \zeta^{2} \sin \theta_{\zeta} \mathrm{d} \theta_{\zeta} \mathrm{d} \zeta
$$

They are functions of $r$ only. The other components of the flow velocity, stress tensor, and heat-flow vector all vanish, i.e., $u_{\theta}^{\alpha}=u_{\varphi}^{\alpha}=0, P_{r \theta}^{\alpha}=P_{r \varphi}^{\alpha}=P_{\theta \varphi}^{\alpha}=0$, and $Q_{\theta}^{\alpha}=Q_{\varphi}^{\alpha}=0$. The macroscopic quantities of the total mixture are written in terms of those of the component gases as

$$
\begin{aligned}
& \omega=\frac{\omega^{A}+\hat{n}_{0}^{B} \omega^{B}}{1+\hat{n}_{0}^{B}}, \quad u_{r}=\frac{u_{r}^{A}+\hat{m}^{B} \hat{n}_{0}^{B} u_{r}^{B}}{1+\hat{m}^{B} \hat{n}_{0}^{B}}, \\
& \tau=\frac{\tau^{A}+\hat{n}_{0}^{B} \tau^{B}}{1+\hat{n}_{0}^{B}}, \quad P=\omega+\tau, \\
& P_{r r}=\frac{P_{r r}^{A}+\hat{n}_{0}^{B} P_{r r}^{B}}{1+\hat{n}_{0}^{B}}, \quad P_{\theta \theta}=P_{\varphi \varphi}=\frac{3 P-P_{r r}}{2},
\end{aligned}
$$

$$
Q_{r}=Q_{r}^{*}+\frac{5}{2}\left(u_{r}^{*}-u_{r}\right)
$$

Here $u_{r}^{*}$ and $Q_{r}^{*}$ in Eq. (10d), which are introduced for the later convenience, are defined by

$$
u_{r}^{*}=\frac{u_{r}^{A}+\hat{n}_{0}^{B} u_{r}^{B}}{1+\hat{n}_{0}^{B}}, \quad Q_{r}^{*}=\frac{Q_{r}^{A}+\hat{n}_{0}^{B} Q_{r}^{B}}{1+\hat{n}_{0}^{B}} .
$$

That is, $u_{r}^{*}$ (or $Q_{r}^{*}$ ) is the average of $u_{r}^{\alpha}$ (or $Q_{r}^{\alpha}$ ) of individual component gases weighted by the reference number density $n_{0}^{\alpha}$.

\section{Conservation relations}

Let us derive the conservation relations from the basic equation and the boundary condition. Integrating Eq. (2) multiplied by $\zeta^{2} \sin \theta_{\zeta} E^{\alpha}$ over the whole domain of $\theta_{\zeta}$ and $\zeta$ (i.e., $0 \leq \theta_{\zeta} \leq \pi$ and $0 \leq \zeta<\infty$ ) and taking into account the boundary condition (4b) for gas $B$, we obtain the following relations describing the mass conservation of each component:

$$
\begin{aligned}
& r^{2} u_{r}^{A}=\frac{M_{f}}{8 \pi L^{2} p_{0}^{A}\left(2 \kappa T_{0} / m^{A}\right)^{-1 / 2}}, \\
& u_{r}^{B}=0 .
\end{aligned}
$$

Here $M_{f}$ is a constant equal to the total mass of the vapor evaporating from the droplet per unit time (the evaporation rate or the mass-flow rate). Equation (12b) means that the noncondensable gas does not have flow velocities in the whole space as a result of the diffuse reflection condition (4b). Substituting Eq. (12b) into the definitions of $u_{r}$ and $u_{r}^{*}$ [Eqs. (10a) and (11)], we have

$$
u_{r}=\frac{u_{r}^{A}}{1+\hat{m}^{B} \hat{n}_{0}^{B}}, \quad u_{r}^{*}=\frac{u_{r}^{A}}{1+\hat{n}_{0}^{B}} .
$$

Similarly, if we integrate Eq. (2) multiplied by $\hat{n}_{0}^{\alpha} \hat{m}^{\alpha} \zeta^{3} \sin 2 \theta_{\zeta} E^{\alpha}$ or $\hat{n}_{0}^{\alpha} \hat{m}^{\alpha} \zeta^{4} \sin \theta_{\zeta} E^{\alpha}$ over the whole domain of $\theta_{\zeta}$ and $\zeta$, and take the summation with respect to $\alpha$ (i.e., $\alpha=A$ and $B$ ), then we obtain the following relations describing the conservation of momentum or energy of the total mixture:

$$
\begin{aligned}
& r^{3} P_{r r}=P_{r r}(r=1)+3 \int_{1}^{r} t^{2} P(t) \mathrm{d} t, \\
& r^{2}\left(Q_{r}+\frac{5}{2} u_{r}\right)=\frac{E_{f}}{4 \pi L^{2} p_{0}\left(2 \kappa T_{0} / m^{A}\right)^{1 / 2}},
\end{aligned}
$$

where $E_{f}$ is a constant equal to the total energy flowing from the droplet to the gas per unit time (the energy-flow rate). The above relations (12) and (14) will be used later to estimate the accuracy of numerical computations. 


\section{ANALYSIS OF THE PROBLEM}

\section{A. Decomposition of the solution and Onsager's reciprocity relation}

Since $\hat{m}^{A}=\hat{K}^{A A}=\hat{n}_{0}^{A}=1$ by definition, the boundary-value problem [Eqs. (2), (4), and (7)] is characterized by seven parameters, i.e., $\hat{m}^{B}, \hat{K}^{B B}, \hat{K}^{A B}, \hat{n}_{0}^{B}, k, \Delta P$, and $\Delta T$. Because of the linearity of the problem, however, the solution for arbitrary values of $\Delta P$ and $\Delta T(\ll 1)$ may be decomposed into two parts as

$$
\Phi^{\alpha}\left(r, \zeta, \theta_{\zeta}\right)=\Phi_{P}^{\alpha}\left(r, \zeta, \theta_{\zeta}\right) \Delta P+\Phi_{T}^{\alpha}\left(r, \zeta, \theta_{\zeta}\right) \Delta T,
$$

where $\Phi_{P}^{\alpha}$ is the solution for $(\Delta P, \Delta T)=(1,0)$ and $\Phi_{T}^{\alpha}$ that for $(\Delta P, \Delta T)=(0,1)$. It should be noted here that the existence of the two independent solutions $\Phi_{P}^{\alpha}$ and $\Phi_{T}^{\alpha}$ (or equivalently the existence of the solution $\Phi^{\alpha}$ for arbitrary $\Delta P$ and $\Delta T$ ) is not guaranteed in advance, but will be confirmed in the subsequent analyses where $\Phi_{P}^{\alpha}$ and $\Phi_{T}^{\alpha}$ will actually be obtained numerically or analytically. The existence of $\Phi_{P}^{\alpha}$ and $\Phi_{T}^{\alpha}$ is due to the fact that the problem concerns a gas around a finite body in the three-dimensional space. See the discussion in Ref. 28 in the case of a single-component gas.

The macroscopic quantities $h\left(h=\omega^{\alpha}, \omega, u_{r}^{\alpha}, u_{r}\right.$, etc. $)$ are correspondingly expressed as

$$
h(r)=h_{P}(r) \Delta P+h_{T}(r) \Delta T .
$$

Here, $h_{J}(J=P, T)$ represents the macroscopic quantities of gas $\alpha$ corresponding to $\Phi_{J}^{\alpha}$ [i.e., Eq. (8) with $\Phi^{\alpha}$ being replaced by $\Phi_{J}^{\alpha}$ ] or those counterparts of the total mixture [i.e., Eq. (10) with $h$ being replaced by $h_{J}$ ].

Let us now introduce some important results derived from Onsager's reciprocity relation and the principle of increase in entropy. Sharipov ${ }^{56,57}$ extended the applicability of Onsager's relation to open gaseous system described by the linearized Boltzmann equation at any rarefaction (or at any Knudsen numbers). His results are applicable also to the present analysis based on the model equation. Following the discussion in Ref. 57, one can derive the following expression for the entropy production $\sigma$ in the system considered in the present problem (see also Ref. 42):

$$
\frac{\sigma}{\kappa / m^{A}}=\frac{8 \pi L^{2} p_{0}}{\left(2 \kappa T_{0} / m^{A}\right)^{1 / 2}}\left(r^{2} u_{r}^{*} \Delta P+r^{2} Q_{r}^{*} \Delta T\right) .
$$

Note that $r^{2} u_{r}^{*}$ and $r^{2} Q_{r}^{*}$ are constants with respect to $r$ [see Eqs. (12a), (13), (14b), and (10d)]. Since $r^{2} u_{r}^{*}$ and $r^{2} Q_{r}^{*}$, which correspond to what is called (dimensionless) thermodynamic fluxes conjugate to the thermodynamic forces $\Delta P$ and $\Delta T$, are also expressed in the form of Eq. (16), Onsager's reciprocity relation may be written as

$$
r^{2} Q_{r P}^{*}=r^{2} u_{r T}^{*}
$$

Here, we should note that the discussion in Ref. 56 on the asymptotic behavior of the velocity distribution function at infinity is not correct. This fact was pointed out by Takata $^{58,59}$ recently. In the present problem, we can verify that the error does not affect the final relation (18). Furthermore, because of the principle of increase in entropy (i.e., $\sigma \geq 0$ ), one has

$$
\begin{aligned}
& r^{2} u_{r P}^{*} \geq 0, \quad r^{2} Q_{r T}^{*} \geq 0, \\
& \left(r^{2} u_{r P}^{*}\right)\left(r^{2} Q_{r T}^{*}\right)-\left(r^{2} Q_{r P}^{*}\right)\left(r^{2} u_{r T}^{*}\right) \geq 0 .
\end{aligned}
$$

The above relations will be referred to in the later discussion.

\section{B. Free-molecular solution for $k \rightarrow \infty$ and asymptotic solution for $k \ll 1$}

We first present the solutions for the free-molecular gas (or the Knudsen gas) in the limit $k \rightarrow \infty$. Since the collision term [i.e., the right-hand side (RHS)] of Eq. (2) vanishes in this limit, the velocity distribution function $\Phi^{\alpha}$ does not change along the characteristic, i.e., $r \sin \theta_{\zeta}=$ const. Therefore, the solutions $\Phi_{P}^{\alpha}$ and $\Phi_{T}^{\alpha}$ satisfying the boundary conditions (4) and (7) are readily obtained and the corresponding macroscopic quantities can be calculated from Eq. (8) as follows:

$$
\begin{aligned}
& \Phi_{P}^{A}= \begin{cases}1 & {\left[0 \leq \theta_{\zeta}<\sin ^{-1}(1 / r)\right],} \\
0 & {\left[\sin ^{-1}(1 / r)<\theta_{\zeta} \leq \pi\right],}\end{cases} \\
& \Phi_{P}^{B}=0, \\
& \omega_{P}^{A}=P_{P}^{A}=\frac{1}{2}\left[1-\left(1-r^{-2}\right)^{1 / 2}\right], \\
& \tau_{P}^{A}=0, \quad P_{r r P}^{A}=\frac{1}{2}\left[1-\left(1-r^{-2}\right)^{3 / 2}\right], \\
& u_{r P}^{A}=-2 Q_{r P}^{A}=\frac{\pi^{-1 / 2}}{2} r^{-2}, \\
& \omega_{P}^{B}=P_{P}^{B}=\tau_{P}^{B}=P_{r r P}^{B}=Q_{r P}^{B}=0,
\end{aligned}
$$

and

$$
\begin{aligned}
& \Phi_{T}^{A}= \begin{cases}\zeta^{2}-\frac{5}{2} & {\left[0 \leq \theta_{\zeta}<\sin ^{-1}(1 / r)\right],} \\
0 & {\left[\sin ^{-1}(1 / r)<\theta_{\zeta} \leq \pi\right],}\end{cases} \\
& \Phi_{T}^{B}= \begin{cases}\hat{m}^{B} \zeta^{2}-2 & {\left[0 \leq \theta_{\zeta}<\sin ^{-1}(1 / r)\right],} \\
0 & {\left[\sin ^{-1}(1 / r)<\theta_{\zeta} \leq \pi\right],}\end{cases} \\
& -\omega_{T}^{A}=\tau_{T}^{A}=\frac{1}{2}\left[1-\left(1-r^{-2}\right)^{1 / 2}\right], \\
& P_{T}^{A}=P_{r r T}^{A}=0, \quad-u_{r T}^{A}=\frac{2}{9} Q_{r T}^{A}=\frac{\pi^{-1 / 2}}{4} r^{-2},
\end{aligned}
$$




$$
Q_{r T}^{B}=\left(\pi \hat{m}^{B}\right)^{-1 / 2} r^{-2} .
$$

Since the molecular collision is absent in this limit, the amount of gas $B$ (or $\hat{n}_{0}^{B}$ ) and the parameter $\hat{K}^{\alpha \beta}$ have no influence on the behavior of individual component gas. Note that the above results satisfy Eqs. (12b), (14a), (18), and (19) derived in Secs. II D and III A.

Let us next consider the case of small $k$. The present problem for $k \ll 1$ has already been investigated in Ref. 50 with the aid of the asymptotic theory ${ }^{60}$ (see also Refs. 5 and 6). For the sake of brevity, only a part of the results are presented below, i.e., the expressions for the flow velocity $u_{r J}^{A}$ of the vapor and those for the average heat flow $Q_{r J}^{*}(J$ $=P, T)$ defined in Eq. (11) valid up to order $O\left(k^{3}\right)$ [see Ref. 50 for the behavior of other macroscopic quantities as well as of the velocity distribution function (several macroscopic quantities in the limit $k \rightarrow 0$ will be shown later in Sec. IV B)]:

$$
\begin{aligned}
& r^{2} u_{r P}^{A}=\frac{k \Lambda\left(1+k S_{4}\right)}{S}, \quad r^{2} u_{r T}^{A}=-\frac{k^{2} \Lambda S_{2}}{S}, \\
& r^{2} Q_{r P}^{*}=-\frac{5\left(1+\Gamma \hat{n}_{0}^{B} / \hat{m}^{B}\right)}{4\left(1+\hat{n}_{0}^{B}\right)} \frac{k^{2} \Lambda S_{3}}{S}, \\
& r^{2} Q_{r T}^{*}=\frac{5\left(1+\Gamma \hat{n}_{0}^{B} / \hat{m}^{B}\right)}{4\left(1+\hat{n}_{0}^{B}\right)} \frac{k\left(1+k \Lambda S_{1}\right)}{S},
\end{aligned}
$$

with

$$
\begin{aligned}
& S_{1}=-C_{4}^{A}+2 k\left(2 C_{6}^{A}+C_{7}^{A}\right), \quad S_{2}=C_{1}^{A}+2 k C_{5}^{A}, \\
& S_{3}=-d_{4}^{M}+2 k\left(2 d_{6}^{M}+d_{7}^{M}\right), \quad S_{4}=d_{1}^{M}+2 k d_{5}^{M}, \\
& S=1+k\left(\Lambda S_{1}+S_{4}\right)+k^{2} \Lambda\left(S_{1} S_{4}-S_{2} S_{3}\right),
\end{aligned}
$$

and

$$
\begin{aligned}
& \Lambda=\frac{\left(1+\hat{K}^{A B} \hat{n}_{0}^{B}\right)\left(1+\hat{m}^{B}\right)}{2 \hat{K}^{A B} \hat{n}_{0}^{B} \hat{m}^{B}}, \\
& \Gamma=\frac{1+\hat{K}^{A B} \hat{n}_{0}^{B}}{\hat{K}^{A B}+\hat{K}^{B B} \hat{n}_{0}^{B}} .
\end{aligned}
$$

Here $C_{i}^{A}$ and $d_{i}^{M}(i=1,4,5,6,7)$ are constants, the so-called jump coefficients, which are relevant to the pressure or temperature jump occurring on the surface of the condensed phase. Those jump coefficients are determined by analyzing the behavior of the mixture in a thin layer, whose thickness is of the order of the molecular mean free path, adjacent to the surface, i.e., by solving the Knudsen-layer problem formulated in Sec. 4 in Ref. 50. In the case of the model equation, $C_{i}^{A}$ and $d_{i}^{M}$ depend on four parameters: $\hat{m}^{B}, \hat{K}^{B B}, \hat{K}^{A B}$, and $\hat{n}_{0}^{B}$. Their data in the case of $\hat{K}^{B B}=\hat{K}^{A B}=1$ are given in Ref. 50 for several values of $\hat{m}^{B}$ and $\hat{n}_{0}^{B}$.

Taking the limit $\hat{n}_{0}^{B} \rightarrow 0$ of Eq. (22), one obtains the following results for a pure vapor:

$$
\begin{aligned}
& r^{2} u_{r P}^{A}=-\frac{1}{C_{4}^{A}}, \quad r^{2} u_{r T}^{A}=\frac{C_{1}^{A}}{C_{4}^{A}} k, \\
& r^{2} Q_{r P}^{A}=-\frac{5}{4} \frac{d_{4}^{M}}{C_{4}^{A}} k, \quad r^{2} Q_{r T}^{A}=\frac{5}{4} k .
\end{aligned}
$$

The above expression for $r^{2} u_{r P}^{A}$ is valid up to $O(1)$ and those for the others are up to $O(k)$. The values of coefficients for $\hat{n}_{0}^{B}=0$ are $-1 / C_{4}^{A}=0.469035, C_{1}^{A} / C_{4}^{A}=-0.261926$, and $d_{4}^{M} / C_{4}^{A}=0.209541$ (see Ref. 50). Comparing Eqs. (22) and (25), we note that $r^{2} u_{r P}^{A}$ vanishes as $k \rightarrow 0$ in the case of a mixture $\left(\hat{n}_{0}^{B} \neq 0\right)$ but does not in the case of a pure vapor $\left(\hat{n}_{0}^{B}=0\right)$.

It is finally noted that by using the data of the jump coefficients in Ref. 50 (for $\hat{K}^{B B}=\hat{K}^{A B}=1$ ), one sees that Eq. (22) [or Eq. (25)] incidentally satisfies Onsager's relation (18) up to $O\left(k^{2}\right)$ [or up to $O(k)$ ] quite well. The inequalities (19) are satisfied for sufficiently small $k$.

\section{Numerical analysis for intermediate values of $\boldsymbol{k}$}

In the case of intermediate values of $k$, we carry out direct numerical analyses of the boundary-value problem for $\Phi_{P}^{\alpha}$ and $\Phi_{T}^{\alpha}$ [i.e., Eqs. (2), (4), and (7) with $(\Delta P, \Delta T)$ $=(1,0)$ and those with $(\Delta P, \Delta T)=(0,1)]$ by means of a finite-difference method. The difference scheme for Eq. (2) and iteration process for obtaining the solution are essentially the same as those described in Ref. 28 , where the present problem in the case of a single-component system (i.e., $\hat{n}_{0}^{B}=0$ ) is analyzed on the basis of the original Boltzmann equation. However, since we are now handling much simpler model equation, the computation of the collision term is much easier. In the following, we just summarize some difficulties which we still share with Ref. 28. The lattice system for $\left(r, \zeta, \theta_{\zeta}\right)$ and the criterion for convergence employed in the present computation will be given later in Sec. IV C.

The first difficulty comes from the fact that the velocity distribution function generally exhibits discontinuities around convex bodies. ${ }^{25,52}$ In the present problem around the spherical droplet, the discontinuity exists at $\theta_{\zeta}=\pi / 2$ on the surface of the droplet $(r=1)$ and propagates into the gas along the characteristic of Eq. (2) touching the droplet, i.e.,

$$
r \sin \theta_{\zeta}=1 \quad\left(0 \leq \theta_{\zeta} \leq \pi / 2\right) .
$$

The discontinuity decays rapidly by molecular collisions [except for the case of the free-molecular gas; see Eqs. (20) and (21)] but is appreciable at least in the vicinity of the droplet. If one approximates the derivatives $\partial \Phi^{\alpha} / \partial r$ and $\partial \Phi^{\alpha} / \partial \theta_{\zeta}$ in the neighborhood of characteristic (26) by taking differences in $\Phi^{\alpha}$ across the discontinuity, unacceptable errors may be introduced. To bypass this difficulty, we employ the hybrid scheme devised in Ref. 25, by which the discontinuity is properly handled. In this scheme, values of $\Phi^{\alpha}$ on both sides of the discontinuity are calculated by finite differences along the characteristic separately, and then are embedded in an ordinary scheme on a rectangular lattice system in the $\left(r, \theta_{\zeta}\right)$-plane. The same method has been successfully applied to various flow problems around a cylindrical or spherical 
body in Refs. 26-28, 32, and 61. The discontinuity should be also taken into account in the numerical integrations of Eq. (8).

The second difficulty comes from the fact that $\Phi^{\alpha}$ for large $r$ approaches to zero very slowly $\left(\sim r^{-n}\right)$ in the present problem, which can be expected from the free-molecular solution (for $k \rightarrow \infty$ ) and the asymptotic solution for small $k$ shown in the previous section. In the numerical analyses, therefore, we need vast computational domain $1 \leq r \leq r_{D}$ with extremely large $r_{D}$, if we directly apply the boundary condition (7) at $r=r_{D}$. Instead, to reduce the size of the computational domain without spoiling the accuracy, we apply the following connection condition at $r=r_{D}$ with reasonably large $r_{D}$ :

$$
\Phi^{\alpha}\left(r_{D}, \zeta, \theta_{\zeta}\right)=\Phi_{\text {far }}^{\alpha}\left(r_{D}, \zeta, \theta_{\zeta}\right),
$$

where $\Phi_{\text {far }}^{\alpha}$ is the asymptotic solution in the far field from the droplet (i.e., the asymptotic solution for large $r$ ). The explicit form of $\Phi_{\text {far }}^{\alpha}$ is given in Appendix A. This technique was proposed and applied successfully in previous studies (e.g., Refs. 27, 28, and 61). As for the molecular velocity $\zeta$, we use likewise a finite computational domain $0 \leq \zeta \leq \zeta_{D}$ with sufficiently large $\zeta_{D}$. No serious problem is caused in this case since $\Phi^{\alpha} E^{\alpha}$ decays exponentially as $\zeta \rightarrow \infty$. The values of $r_{D}$ and $\zeta_{D}$ used in the computation will be given later in Sec. IV C.

In addition to the countermeasures against the difficulties mentioned above, we also adopt the idea in Refs. 27 and 61 to improve the accuracy of computations for small Knudsen numbers. That is, in the case of small $k$ (say, $k \leqq 0.1$ ), we carry out computations not for $\Phi^{\alpha}$ directly but for $\widetilde{\Phi}^{\alpha}\left(\equiv \Phi^{\alpha}-\Phi_{H[1]}^{\alpha}\right)$, where $\Phi_{H[1]}^{\alpha}$ is the Hilbert solution up to $O(k)$ obtained in the asymptotic analysis in Ref. 50 (i.e., $\Phi_{H[1]}^{\alpha}$ here corresponds to $\varphi_{H 0}^{\alpha}+\varphi_{H 1}^{\alpha} k$ in that reference). The numerical computations for $\widetilde{\Phi}^{\alpha}$ might be more advantageous than those for $\Phi^{\alpha}$ in the case of small $k$ since $\widetilde{\Phi}^{\alpha}=O\left(k^{2}\right)$ [or $\widetilde{\Phi}^{\alpha}=O(k)$ in the Knudsen layer adjacent to droplet's surface] and the relative errors occurring in the computations for $\widetilde{\Phi}^{\alpha}$ and those for $\Phi^{\alpha}$ are expected to be of the same order (see the discussion in Refs. 27 and 61).

\section{RESULTS AND DISCUSSIONS}

In the present paper, we limit ourselves to the case of $\hat{m}^{B}=0.5,1$, and $2, \hat{n}_{0}^{B}=0.1,1$, and 10 , and $\hat{K}^{B B}=\hat{K}^{A B}=1$. Note that $\hat{m}^{B}=1$ (with $\hat{K}^{B B}=\hat{K}^{A B}=1$ ) corresponds to the case where the molecules of the vapor and those of the noncondensable gas are mechanically identical. For each pair of $\hat{m}^{B}$ and $\hat{n}_{0}^{B}$, numerical computations are carried out for $k=20,10,5,2,1$, $0.5,0.2,0.1$, and 0.05 . Those numerical results, together with the results based on the analytical solutions for large and small $k$, are presented in this section.

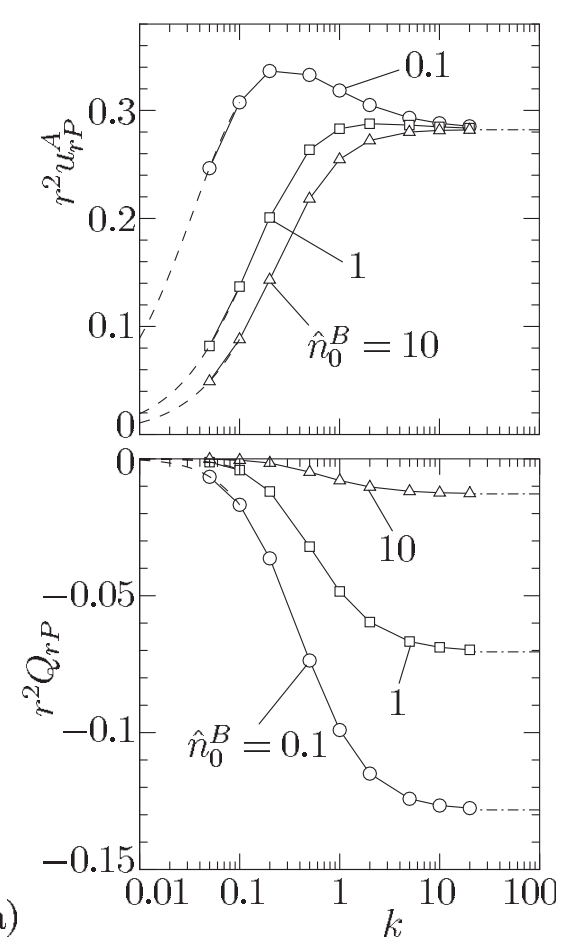

(a)

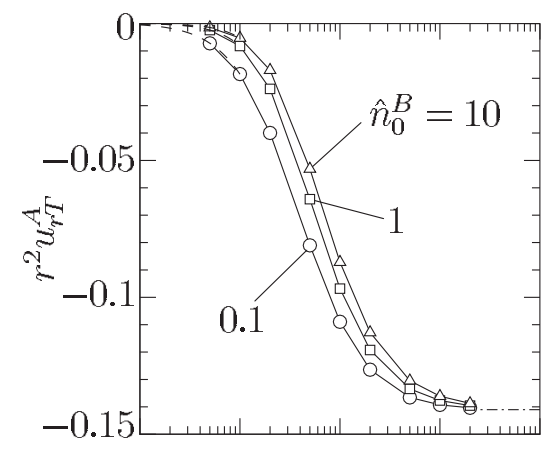

(b)

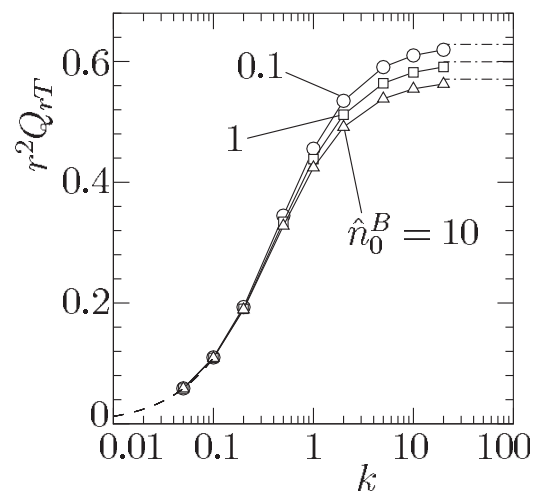

FIG. 1. The mass- and heat-flow rates vs $k$ for $\hat{m}^{B}=1$. (a) $r^{2} u_{r P}^{A}$ and $r^{2} Q_{r P}$ and (b) $r^{2} u_{r T}^{A}$ and $r^{2} Q_{r T}$. The symbols indicate numerical results for $\hat{n}_{0}^{B}=0.1(\bigcirc), 1(\square)$, and $10(\triangle)$. The asymptotic solution for small $k$ in Ref. 50 is represented by dashed lines and the free-molecular solution for $k \rightarrow \infty$ by dashed-dotted lines.

\section{A. Mass- and heat-flow rates}

The dimensionless mass-flow rate (or the evaporation rate) $r^{2} u_{r J}^{A}$ and the heat-flow rate $r^{2} Q_{r J}(J=P, T)$ are shown as functions of $k$ in Figs. 1-3; Fig. 1 is for $\hat{m}^{B}=1$, Fig. 2 for $\hat{m}^{B}=0.5$, and Fig. 3 for $\hat{m}^{B}=2$. See supplementary material ${ }^{62}$ for tables of their numerical data. While $r^{2} u_{r J}^{A}$ and $r^{2} Q_{r J}$ are constant with respect to $r$ theoretically (see Sec. II D), their 


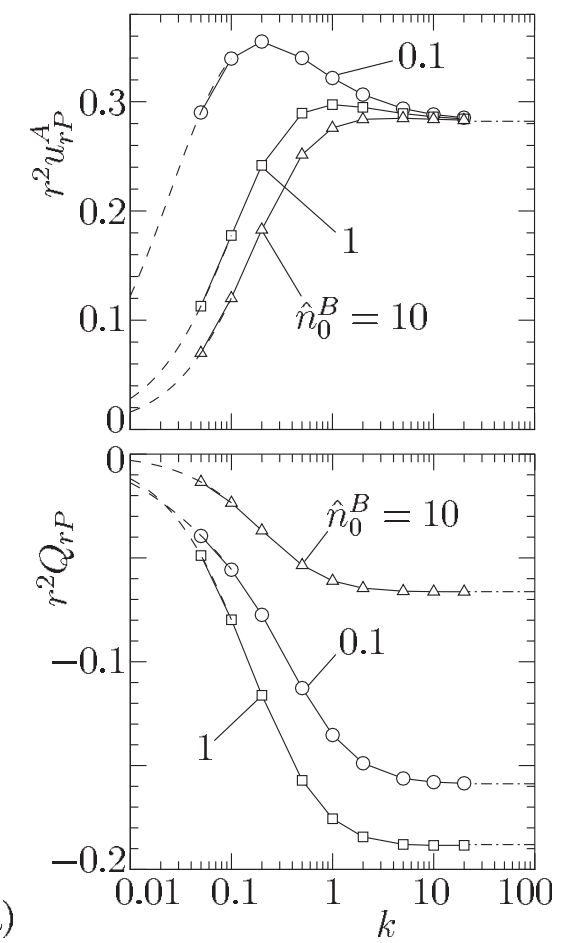

(a)

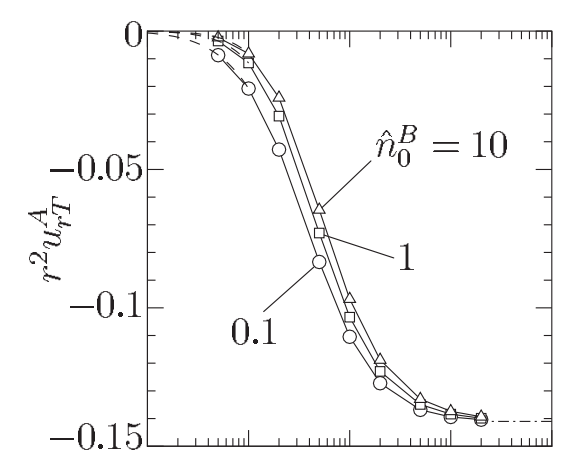

(b)

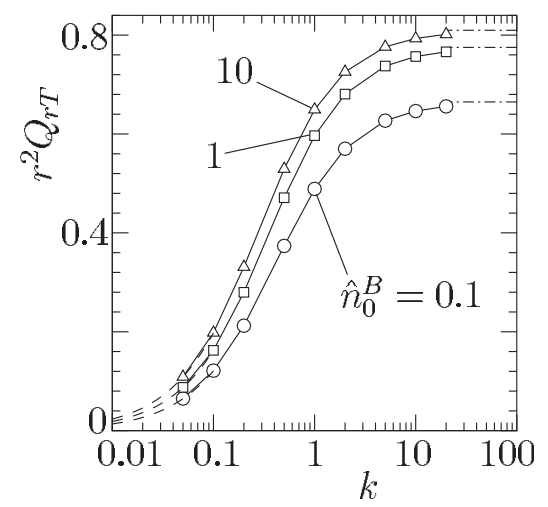

FIG. 2. The mass- and heat-flow rates vs $k$ for $\hat{m}^{B}=0.5$. (a) $r^{2} u_{r P}^{A}$ and $r^{2} Q_{r P}$ and (b) $r^{2} u_{r T}^{A}$ and $r^{2} Q_{r T}$. See the caption of Fig. 1.

values obtained in the numerical analyses are not exactly uniform but show small fluctuations caused by numerical errors (the magnitude of the fluctuations will be given later in Sec. IV C). Their values at $r=1$ are, therefore, shown as representatives in the figures and tables. The results for the free-molecular gas [Eqs. (20) and (21)] and for small $k$ [Eq. (22)] are also shown in the figures.

We first consider the mass-flow rates $r^{2} u_{r P}^{A}$ and $r^{2} u_{r T}^{A}$. As seen in the figures, $r^{2} u_{r P}^{A}>0$ and $r^{2} u_{r T}^{A}<0$ hold irrespective
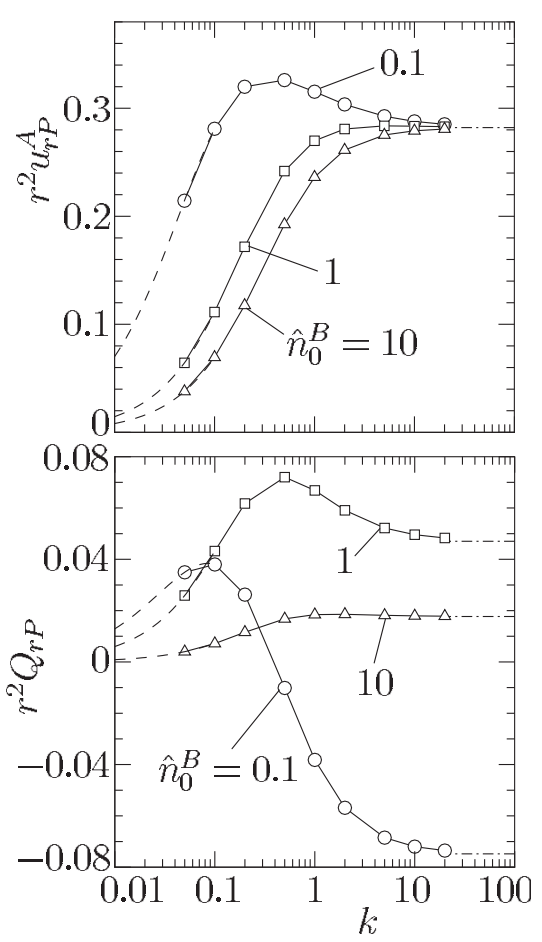

(a)

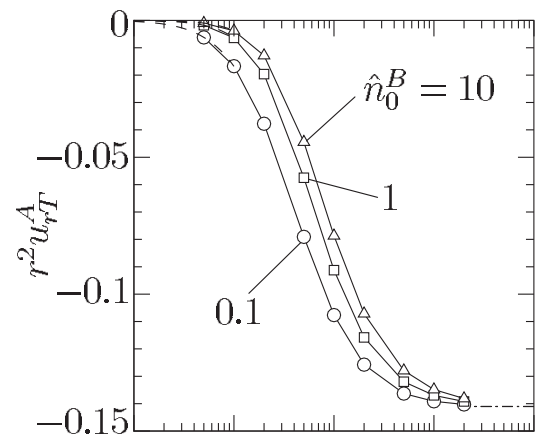

(b)

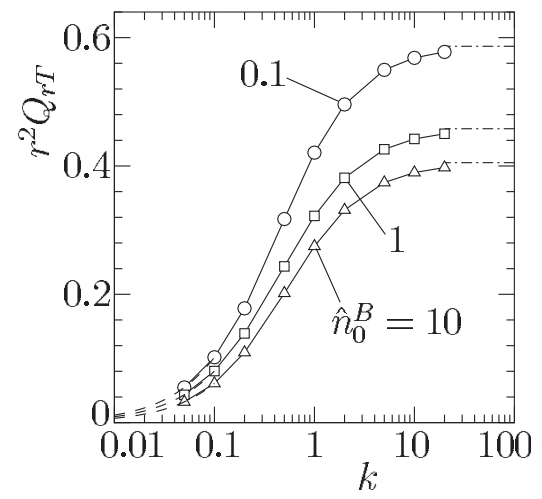

FIG. 3. The mass- and heat-flow rates vs $k$ for $\hat{m}^{B}=2$. (a) $r^{2} u_{r P}^{A}$ and $r^{2} Q_{r P}$ and (b) $r^{2} u_{r T}^{A}$ and $r^{2} Q_{r T}$. See the caption of Fig. 1.

of the values of $k$ and $\hat{n}_{0}^{B}$. The former corresponds to the evaporation, which is consistent with the first inequality of Eq. (19a), and the latter to the condensation. The $r^{2} u_{r P}^{A}$ and $r^{2} u_{r T}^{A}$ approach their limiting values for the free-molecular gas, which are independent of $\hat{n}_{0}^{B}$ [see the sentence just after Eq. (21)], as $k \rightarrow \infty$, and vanish as $k \rightarrow 0$ [see Eq. (22)]. Since the noncondensable gas cannot flow, i.e., $u_{r}^{B} \equiv 0$ [see Eq. (12b)], in the present problem, it just obstructs the vapor flow and such obstruction is expected to be stronger for 
larger values of $\hat{m}^{B}$ and $\hat{n}_{0}^{B}$. The absolute values of $r^{2} u_{r P}^{A}$ and $r^{2} u_{r T}^{A}$ for fixed $k$ indeed become smaller for larger $\hat{m}^{B}$ or $\hat{n}_{0}^{B}$. However, the dependence of $r^{2} u_{r T}^{A}$ on $\hat{m}^{B}$ and $\hat{n}_{0}^{B}$ is relatively weak. The magnitude of $r^{2} u_{r T}^{A}$ is monotonically increasing as a function of $k$, whereas $r^{2} u_{r P}^{A}$ may have a peak at an intermediate value of $k$ as seen from the figures [see the data for $\hat{n}_{0}^{B}=0.1$ in Figs. 1(a), 2(a), and 3(a)]. The nonmonotonic behavior of $r^{2} u_{r P}^{A}$ will be discussed again in a later paragraph in this section.

As for the heat-flow rates $r^{2} Q_{r P}$ and $r^{2} Q_{r T}$, they show strong and complicated dependence on $\hat{m}^{B}$ and $\hat{n}_{0}^{B}$. In particular, $r^{2} Q_{r P}$ for $\hat{m}^{B}=2$ shows nonmonotonic behavior: It is positive in the entire range of $k$ in the case of $\hat{n}_{0}^{B}=1$ and 10 , but changes its sign depending on $k$ in the case of $\hat{n}_{0}^{B}=0.1$. The $r^{2} Q_{r T}$ for fixed $k$ becomes larger for smaller (or larger) values of $\hat{n}_{0}^{B}$ in the case of $\hat{m}^{B}=1$ and 2 (or in the case of $\hat{m}^{B}=0.5$ ). The heat-flow rates vanish as $k \rightarrow 0$ [see Eq. (22)].

We now make a comparison with the results for a pure vapor $\left(\hat{n}_{0}^{B}=0\right)$ in Ref. 28 . The behavior (i.e., the dependence on $k$ ) of $r^{2} Q_{r P}, r^{2} u_{r T}^{A}$, and $r^{2} Q_{r T}$ for $\hat{n}_{0}^{B}=0.1$ in Figs. 1-3 are similar to that of the corresponding quantities for a pure vapor, i.e., Figs. 1 and 2 in Ref. 28 (except $r^{2} Q_{r P}$ for $\hat{m}^{B}=2$ ). Therefore, their behavior for the mixture seems to approach that for a pure vapor as $\hat{n}_{0}^{B}$ tends to vanish. The behavior of $r^{2} u_{r P}^{A}$ for $\hat{n}_{0}^{B}=0.1$ is, however, qualitatively different from that for a pure vapor $\left(\hat{n}_{0}^{B}=0\right)$ in Ref. 28. More specifically, $r^{2} u_{r P}^{A}$ has a peak at an intermediate value of $k$ (cf. the second paragraph in this section), but the mass-flow rate for a pure vapor increases monotonically as $k$ decreases (cf. Fig. 1 in Ref. 28). The difference can be explained as follows. In the case of $\hat{n}_{0}^{B}=0, r^{2} u_{r P}^{A}$ is still given by Eq. (20e) in the freemolecular limit $(k \rightarrow \infty)$. It increases monotonically with decreasing $k$ and approaches its limit, i.e., Eq. (25a), as $k \rightarrow 0$ (see Fig. 1 in Ref. 28). In the case of $\hat{n}_{0}^{B} \neq 0$, the vapor flow is completely blocked $\left(r^{2} u_{r P}^{A} \rightarrow 0\right)$ in the limit $k \rightarrow 0$ by frequent molecular collisions with gas $B$ [see Eq. (22a)]. If $\hat{n}_{0}^{B}$ is sufficiently large (say $\hat{n}_{0}^{B}=10$ ), the effect of gas $B$ as an obstacle is dominant in the whole range of $k$ and thus $r^{2} u_{r P}^{A}$ decreases monotonically with decreasing $k$. If $\hat{n}_{0}^{B}$ is small (say $\hat{n}_{0}^{B}=0.1$ ), however, the effect of gas $B$ manifests itself only in the small- $k$ region. Therefore, $r^{2} u_{r P}^{A}$ increases with decreasing $k$ in the large- $k$ region as in the case of a pure vapor. The combination of these two effects causes the nonmonotonic behavior of $r^{2} u_{r P}^{A}$. See also Refs. 50 and 42 for discussions on the behavior of $r^{2} u_{r P}^{A}$ for a pure vapor and that for a mixture.

The tables for $r^{2} u_{r J}^{A}$ and $r^{2} Q_{r J}(J=P, T)$ presented in the supplementary material ${ }^{62}$ also show the values of $r^{2}(1$ $\left.+\hat{n}_{0}^{B}\right) Q_{r P}^{*}$. Because of Onsager's reciprocity relation (18), it should coincide with $r^{2} u_{r T}^{A}$ in the whole range of $k$ theoretically [see also Eq. (13)]. Their values in the tables show good agreement, with small differences at small $k$. The differences give an estimate for accuracy of the numerical computations. Note that in the case of $\hat{m}^{B}=1, Q_{r P}=Q_{r P}^{*}$ holds by definition and thus $r^{2} Q_{r P}=r^{2} u_{r T}^{A} /\left(1+\hat{n}_{0}^{B}\right)$ follows. We also confirmed that the numerical solutions satisfy the second inequality of Eq. (19a) and Eq. (19b).

Figure 4 shows the results for $\hat{m}^{B}=1$ of the present computations and those of Young's approximate formula pro- (a)

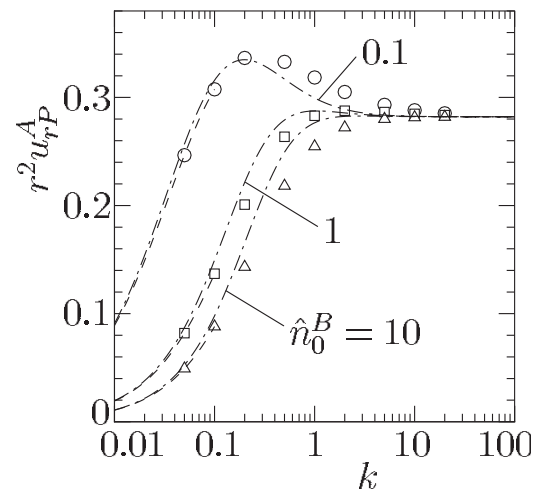

(b)
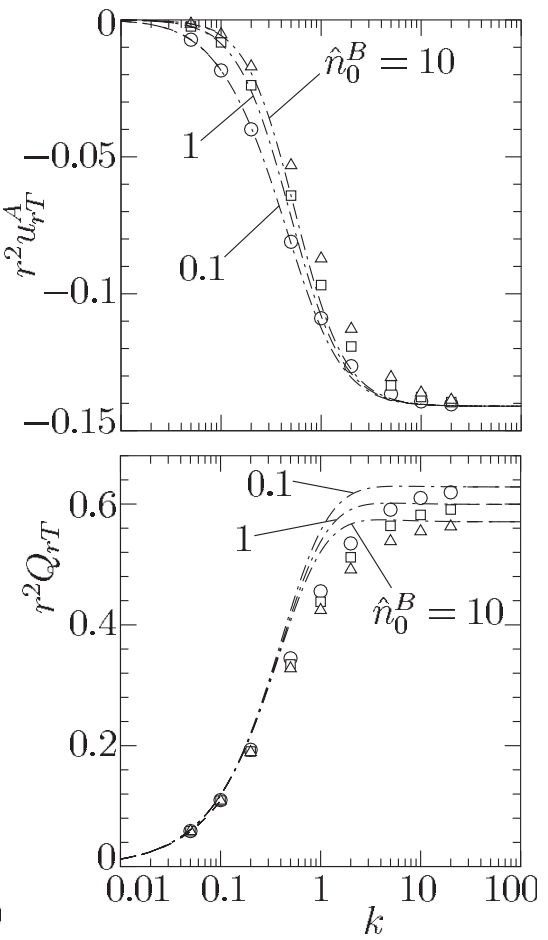

FIG. 4. Comparison with Young's formula (Ref. 42) in the case of $\hat{m}^{B}=1$. (a) $r^{2} u_{r P}^{A}$ and (b) $r^{2} u_{r T}^{A}$ and $r^{2} Q_{r T}$. The symbols indicate present numerical results for $\hat{n}_{0}^{B}=0.1(\bigcirc), 1(\square)$, and $10(\triangle)$, and dashed-dotted lines the results of Young's formula. The dashed lines indicate the asymptotic solution for small $k$ in Ref. 50 or the free-molecular solution for $k \rightarrow \infty$.

posed in Ref. 42. The formula has been constructed on the basis of a simple physical model. However, if the adjustable parameters are chosen appropriately (see the last sentence of Appendix B), it reproduces qualitative features of the present results quite well. We also made comparisons in the case of $\hat{m}^{B}=0.5$ and 2 and arrived at a similar conclusion (see Figs. 1 and 2 in the supplementary material $\left.{ }^{62}\right)$. The $r^{2} Q_{r P}$ is omitted in Fig. 4 because it coincides with $r^{2} u_{r T}^{A} /\left(1+\hat{n}_{0}^{B}\right)$ theoretically (see the last paragraph). The explicit form of Young's formula, as well as some details of the comparison, is presented in Appendix B. By the way, Young's formula has also been verified experimentally in Ref. 63, where excellent agreement between the measurement and the prediction by the formula concerning the droplet growth and evaporation rate is reported.

Finally, let us consider the special case where the present problem reduces to the heat transfer problem. Recalling that 
TABLE I. The heat-flow rate $r^{2} Q_{r} / \Delta T$ and the ratio $\Delta P / \Delta T$ for the heat transfer problem.

\begin{tabular}{|c|c|c|c|c|c|c|}
\hline \multirow[b]{2}{*}{$k$} & \multicolumn{2}{|c|}{$\hat{n}_{0}^{B}=0.1$} & \multicolumn{2}{|c|}{$\hat{n}_{0}^{B}=1$} & \multicolumn{2}{|c|}{$\hat{n}_{0}^{B}=10$} \\
\hline & $\Delta P / \Delta T$ & $r^{2} Q_{r} / \Delta T$ & $\Delta P / \Delta T$ & $r^{2} Q_{r} / \Delta T$ & $\Delta P / \Delta T$ & $r^{2} Q_{r} / \Delta T$ \\
\hline \multicolumn{7}{|c|}{$\hat{m}^{B}=1$} \\
\hline$\infty$ & 0.5 & 0.5642 & 0.5 & 0.5642 & 0.5 & 0.5642 \\
\hline 20 & 0.4920 & 0.5566 & 0.4920 & 0.5566 & 0.4920 & 0.5566 \\
\hline 10 & 0.4834 & 0.5487 & 0.4834 & 0.5487 & 0.4835 & 0.5487 \\
\hline 5 & 0.4659 & 0.5328 & 0.4659 & 0.5328 & 0.4659 & 0.5329 \\
\hline 2 & 0.4145 & 0.4871 & 0.4145 & 0.4871 & 0.4146 & 0.4871 \\
\hline 1 & 0.3421 & 0.4216 & 0.3421 & 0.4216 & 0.3422 & 0.4216 \\
\hline 0.5 & 0.2433 & 0.3268 & 0.2434 & 0.3268 & 0.2434 & 0.3268 \\
\hline 0.2 & 0.1187 & 0.1890 & 0.1188 & 0.1890 & 0.1188 & 0.1890 \\
\hline 0.1 & 0.05998 & 0.1089 & 0.06000 & 0.1089 & 0.06003 & 0.1088 \\
\hline 0.05 & \multicolumn{5}{|c|}{$\hat{m}^{B}=0.5$} & 0.05841 \\
\hline$\infty$ & 0.5 & 0.5854 & 0.5 & 0.6810 & 0.5 & 0.7766 \\
\hline 20 & 0.4920 & 0.5778 & 0.4920 & 0.6734 & 0.4921 & 0.7690 \\
\hline 10 & 0.4835 & 0.5700 & 0.4836 & 0.6656 & 0.4837 & 0.7612 \\
\hline 5 & 0.4660 & 0.5541 & 0.4665 & 0.6498 & 0.4668 & 0.7454 \\
\hline 2 & 0.4151 & 0.5084 & 0.4170 & 0.6038 & 0.4184 & 0.6987 \\
\hline 1 & 0.3434 & 0.4425 & 0.3477 & 0.5357 & 0.3508 & 0.6279 \\
\hline 0.5 & 0.2453 & 0.3460 & 0.2521 & 0.4314 & 0.2569 & 0.5156 \\
\hline 0.2 & 0.1206 & 0.2030 & 0.1271 & 0.2651 & 0.1318 & 0.3263 \\
\hline 0.1 & 0.06122 & 0.1180 & 0.06496 & 0.1572 & 0.06782 & 0.1965 \\
\hline \multicolumn{7}{|c|}{$\hat{m}^{B}=2$} \\
\hline$\infty$ & 0.5 & 0.5492 & 0.5 & 0.4816 & 0.5 & 0.4140 \\
\hline 20 & 0.4920 & 0.5415 & 0.4919 & 0.4739 & 0.4919 & 0.4063 \\
\hline 10 & 0.4834 & 0.5337 & 0.4833 & 0.4661 & 0.4831 & 0.3984 \\
\hline 5 & 0.4658 & 0.5179 & 0.4653 & 0.4503 & 0.4647 & 0.3827 \\
\hline 2 & 0.4142 & 0.4724 & 0.4125 & 0.4060 & 0.4100 & 0.3391 \\
\hline 1 & 0.3415 & 0.4077 & 0.3380 & 0.3448 & 0.3329 & 0.2811 \\
\hline 0.5 & 0.2425 & 0.3148 & 0.2377 & 0.2606 & 0.2309 & 0.2056 \\
\hline 0.2 & 0.1180 & 0.1812 & 0.1143 & 0.1461 & 0.1091 & 0.1106 \\
\hline 0.1 & 0.05957 & 0.1042 & 0.05744 & 0.08290 & 0.05445 & 0.06151 \\
\hline 0.05 & 0.02906 & 0.05583 & 0.02797 & 0.04413 & 0.02646 & 0.03241 \\
\hline
\end{tabular}

the mass-flow rate $r^{2} u_{r}^{A}$ for arbitrary $\Delta P$ and $\Delta T(\ll 1)$ is given as $r^{2} u_{r}^{A}=r^{2} u_{r P}^{A} \Delta P+r^{2} u_{r T}^{A} \Delta T$, one notices that the evaporation or condensation may stop for special values of $\Delta P$ and $\Delta T$ (except the trivial case, i.e., $\Delta P=\Delta T=0$ ) which satisfy

$$
\Delta P / \Delta T=-\left(r^{2} u_{r T}^{A}\right) /\left(r^{2} u_{r P}^{A}\right) .
$$

With such values of $\Delta P$ and $\Delta T$, the boundary condition (4a) at the interface $(r=1)$ for the vapor becomes equivalent to the diffuse reflection condition [i.e., Eq. (4b) with the superscript $B$ being replaced by $A]$. Thus, the corresponding solution $\Phi^{\alpha}$ can be interpreted as a solution for the heat transfer problem between a solid sphere with temperature $T_{w}$ and the surrounding binary mixture which is in a stationary equilibrium state with temperature $T_{0}$ and partial pressures $p_{0}^{A}$ and $p_{0}^{B}$ at infinity. The heat-flow rate $r^{2} Q_{r}\left(=r^{2} Q_{r P} \Delta P+r^{2} Q_{r T} \Delta T\right)$ in such a situation is written as

$$
\begin{aligned}
\frac{r^{2} Q_{r}}{\Delta T} & =\frac{\left(r^{2} u_{r P}^{A}\right)\left(r^{2} Q_{r T}\right)-\left(r^{2} Q_{r P}\right)\left(r^{2} u_{r T}^{A}\right)}{r^{2} u_{r P}^{A}} \\
& =\frac{\left(r^{2} u_{r P}^{*}\right)\left(r^{2} Q_{r T}^{*}\right)-\left(r^{2} Q_{r P}^{*}\right)\left(r^{2} u_{r T}^{*}\right)}{r^{2} u_{r P}^{*}} .
\end{aligned}
$$

The second equality comes from Eqs. (10d) and (13). Because of Eq. (19), $r^{2} Q_{r} / \Delta T$ should be positive. The ratio $\Delta P / \Delta T$ determined by Eq. (28) and the corresponding $r^{2} Q_{r} / \Delta T$ for various $k$ are tabulated in Table I. It is seen from the data in the table and Eq. (22) that both $\Delta P / \Delta T$ and $r^{2} Q_{r} / \Delta T$ decrease with decreasing $k$ and vanish as $k \rightarrow 0$. In the case of $\hat{m}^{B}=1$ (with $\hat{K}^{B B}=\hat{K}^{A B}=1$ ) one can prove theoretically that $\Delta P / \Delta T$ and $r^{2} Q_{r} / \Delta T$ are independent of $\hat{n}_{0}^{B}$, while their values in the table show slight dependence caused by numerical errors. In the case of $\hat{m}^{B}=0.5$ (or $\hat{m}^{B}=2$ ), they increase (or decrease) with increasing $\hat{n}_{0}^{B}$. 

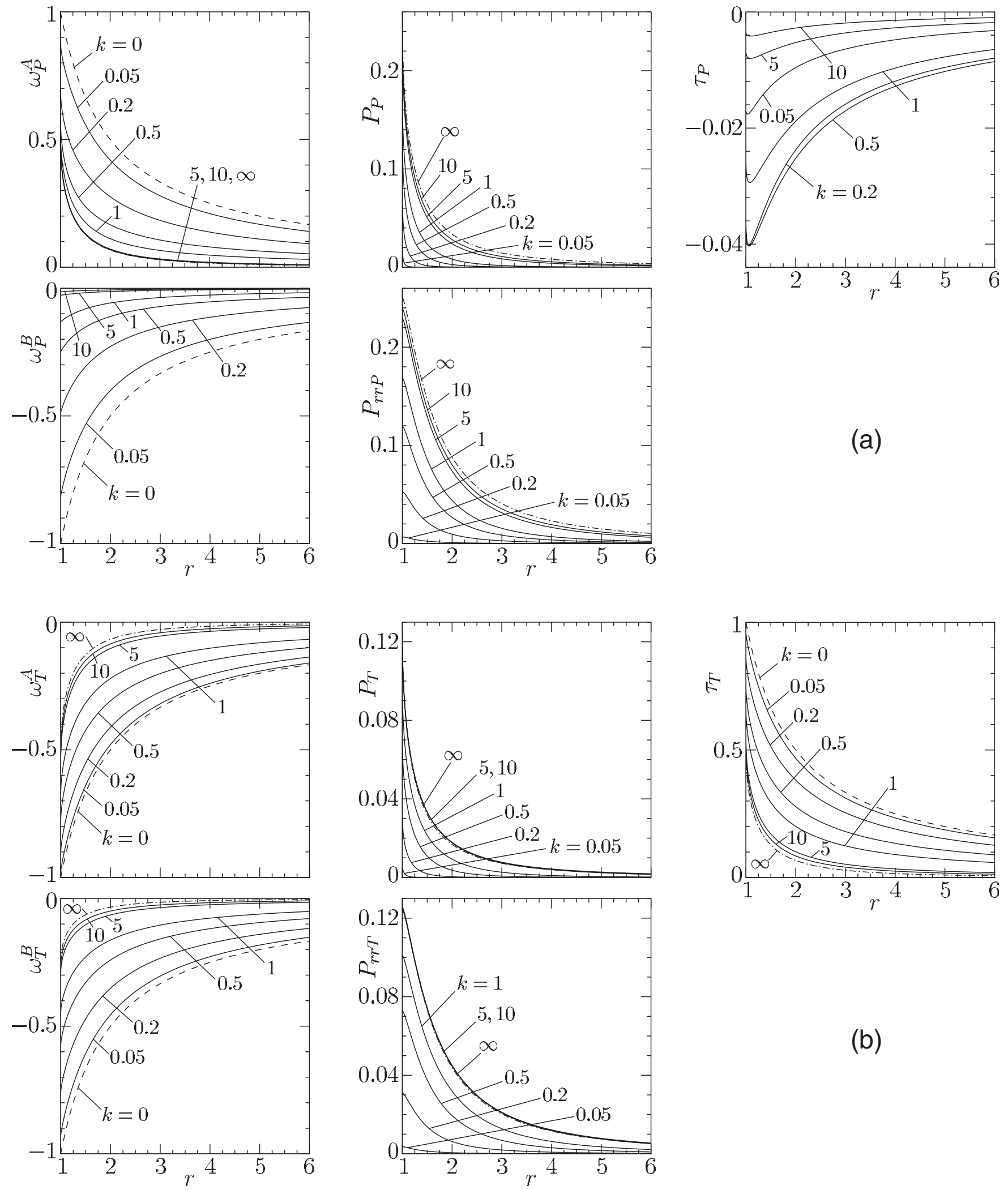

FIG. 5. Profiles of the macroscopic quantities for $\hat{m}^{B}=1$ and $\hat{n}_{0}^{B}=1$. (a) $\omega_{P}^{A}, \omega_{P}^{B}, P_{P}, P_{r r P}$, and $\tau_{P}$ and (b) $\omega_{T}^{A}, \omega_{T}^{B}, P_{T}, P_{r r T}$, and $\tau_{T}$. The dashed lines indicate the results for $k \rightarrow 0$ and dashed-dotted lines those for $k \rightarrow \infty$.

\section{B. Behavior of the macroscopic quantities}

The profiles of the macroscopic quantities for $\hat{m}^{B}=1$ and $\hat{n}_{0}^{B}=1$ are shown in Fig. 5; Fig. 5(a) is for $\omega_{P}^{A}, \omega_{P}^{B}, P_{P}, P_{r r P}$, and $\tau_{P}$ resulting from the solution $\Phi_{P}^{\alpha}$ for $(\Delta P, \Delta T)=(1,0)$, and Fig. 5(b) for $\omega_{T}^{A}, \omega_{T}^{B}, P_{T}, P_{r r T}$, and $\tau_{T}$ from $\Phi_{T}^{\alpha}$ for $(\Delta P, \Delta T)=(0,1)$. The profiles for $k \rightarrow \infty$ [i.e., Eqs. (20) and (21)] and those for $k \rightarrow 0$ derived in Ref. 50 are also shown in the figures $\left(\omega_{P}^{A}=-\hat{n}_{0}^{B} \omega_{P}^{B}=r^{-1}, P_{P}=P_{r r P}=\tau_{P}=0,-\omega_{T}^{A}=-\omega_{T}^{B}\right.$ 
$=\tau_{T}=r^{-1}$, and $P_{T}=P_{r r T}=0$ in the limit $k \rightarrow 0$ ). Also the results for other pairs of $\hat{m}^{B}$ and $\hat{n}_{0}^{B}$ are shown in the supplementary material. $^{62}$

The density $\omega_{P}^{A}$ (or $\omega_{P}^{B}$ ) takes its maximum (or minimum) on the surface of the droplet $(r=1)$ and monotonically decreases (or increases) to zero as $r \rightarrow \infty$. The $\left|\omega_{P}^{B}\right|$ for fixed $r$ is a monotonically decreasing function of $k$ and vanishes as $k \rightarrow \infty$. However, $\omega_{P}^{A}$ does not vanish as $k \rightarrow \infty\left(\omega_{P}^{A}\right.$ for small $\hat{n}_{0}^{B}$ may show slightly nonmonotonic behavior as a function of $k$; see Fig. 3 in the supplementary material ${ }^{62}$ ). The temperature $\tau_{P}$ is negative, takes its minimum in the close vicinity of the droplet (not on the interface), and vanishes as $r \rightarrow \infty$. That is, the mixture is cooler than the droplet and also the equilibrium state at infinity. As a function of $k$, $\tau_{P}$ for fixed $r$ vanishes as $k \rightarrow \infty$ and also as $k \rightarrow 0$, and thus takes its minimum at an intermediate value of $k$ (i.e., $k$ $\sim 0.2-0.5)$. We should note here that $\tau_{P}$ for a pure vapor $\left(\hat{n}_{0}^{B}=0\right)$ also vanishes as $k \rightarrow \infty$ but does not as $k \rightarrow 0$, i.e., $\tau_{P}$ for fixed $r$ monotonically decreases with decreasing $k$ to its limit as $k \rightarrow 0$ (see Fig. 4 in Ref. 28). The different behavior of $\tau_{P}$ for small $k$ is related to the existence of the evaporating flow $u_{r P}^{A}$ for a pure vapor in the limit $k \rightarrow 0$ (see, e.g., Ref. 5, p. 82). The pressure $P_{P}$ and stress tensor $P_{r r P}$ behave monotonically as functions of both $r$ and $k$.

The densities $\omega_{T}^{A}$ and $\omega_{T}^{B}$ and temperature $\tau_{T}$ are monotonic with respect to both $r$ and $k$. The pressure $P_{T}$ and stress tensor $P_{r r T}$ decrease monotonically as functions of $r$, while they may show nonmonotonic behavior as functions of $k$ in the case of $\hat{n}_{0}^{B} \lesssim 1$ (see also Fig. 3 in the supplementary material $\left.^{62}\right)$. Note that in the case of a pure vapor $\left(\hat{n}_{0}^{B}=0\right) P_{T}$ and $P_{r r T}$ vanish as $k \rightarrow \infty$ [see Eq. (21d)].

By comparison with Figs. 3-6 in the supplementary material, ${ }^{62}$ it is seen that the dependence on $\hat{m}^{B}$ is relatively weak and the maximum values of $\left|\omega_{P}^{B}\right|, P_{P}, P_{r r P}$, and $\left|\tau_{P}\right|$ (or $P_{T}$ and $P_{r r T}$ ) decrease (or increase) with increasing $\hat{n}_{0}^{B}$.

\section{Data of computation}

Let us here summarize the data of the lattice system used in the finite-difference analysis and accuracy of the computations for intermediate values of $k$.

In all the computations, we used the following lattice system $\left(r^{(i)}, \zeta^{(j)}, \theta_{\zeta}^{(l)}\right)$ for the independent variables $\left(r, \zeta, \theta_{\zeta}\right)$ :

$$
\begin{aligned}
& r^{(i)}=1+\left(r_{D}-1\right)\left(i / N_{r}\right)^{3} \quad\left(i=0,1, \ldots, N_{r}\right), \\
& \zeta^{(j)}=\zeta_{D}\left(j / N_{\zeta}\right)^{3} \quad\left(j=0,1, \ldots, N_{\zeta}\right), \\
& \theta_{\zeta}^{(l)}=\pi l / 2 N_{\theta} \quad\left(l=0,1, \ldots, 2 N_{\theta}\right),
\end{aligned}
$$

with $N_{r}=600, N_{\zeta}=40, \zeta_{D}=6$, and $N_{\theta}=100$. The $r_{D}$ was set to be proportional to $k$ as $r_{D}=100 k$ for $k \geq 10,150 k$ for $k=5$, and $200 k$ for $k \leq 2$. As mentioned in Sec. III C, the finitedifference scheme for Eq. (2) and iteration process are essentially the same as those in Ref. 28. Also, essentially the same criterion for convergence was used. That is, the iteration was stopped when the variation in the macroscopic variables $\omega^{\alpha}$, $u_{r}^{A}$, and $\tau^{\alpha}$ over the whole space in ten steps becomes less than $1 \times 10^{-8}$. In general, the computations for smaller $k$ need more iterations to achieve the convergence and thus take more CPU time. Typically, about 4.7 days computation using a personal computer with Intel Pentium $43.2 \mathrm{GHz}$ processor was needed to obtain $\Phi_{P}^{\alpha}$ for $\hat{m}^{B}=\hat{n}_{0}^{B}=1$ in the case of $k=0.05$.

As mentioned in the first paragraph in Sec. IV A, $r^{2} u_{r J}^{A}$ and $r^{2} Q_{r J}$ obtained in the numerical analyses are not constant with respect to $r$ but show small fluctuations, which provide a measure of error estimate for the computations. Let $\delta[h(r)]$ be the maximum value of $\left|\left[h\left(r^{(i)}\right)-h(1)\right] / h(1)\right|$ for $1 \leq r^{(i)}$ $\leq 4$, i.e., the maximum relative error of a quantity $h(r)$ evaluated at the lattice points in $1 \leq r \leq 4$. Then, $\delta\left[r^{2} u_{r P}^{A}\right]$ $<0.09 \%$ holds in all the computations, $\delta\left[r^{2} u_{r T}^{A}\right]<0.07 \%$ in the computations for $k \geq 1$, and $\delta\left[r^{2} u_{r T}^{A}\right]<0.24 \%$ in those for $k \leq 0.5$. Meanwhile, $\delta\left[r^{2} Q_{r P}\right]<0.1 \%$ holds for $k \geq 2$ and $\delta\left[r^{2} Q_{r P}\right]<0.58 \%$ for $k \leq 1$ except for the case of $\left(\hat{m}^{B}, \hat{n}_{0}^{B}, k\right)=(2,0.1,0.5)$, where we have $\delta\left[r^{2} Q_{r P}\right]=1.4 \%$; $\delta\left[r^{2} Q_{r T}\right]<0.14 \%$ holds except for the case of $\left(\hat{m}^{B}, \hat{n}_{0}^{B}, k\right)$ $=(0.5,0.1,0.05)$, where we have $\delta\left[r^{2} Q_{r T}\right]=0.52 \%$.

Another measure of accuracy is provided by the flow velocity $u_{r J}^{B}$ of the noncondensable gas, which should be zero theoretically [see Eq. (12b)]. In all the computations, $\left|u_{r P}^{B}\right|$ is less than $6.5 \times 10^{-6}$ and $\left|u_{r T}^{B}\right|$ less than $1.9 \times 10^{-5}$ at the whole lattice points.

\section{EXTENSION TO GENERALIZED BOUNDARY CONDITION}

Up until now, we assumed the complete condensation condition for the vapor [i.e., Eq. (4a)] on the surface of the droplet, where the velocity distribution of outgoing molecules is determined solely by the state of the condensed phase and is not influenced by incoming molecules. In this section, we shall consider a more general boundary condition for the vapor which includes the effect of incoming molecules. The following discussion is the extension of the corresponding one for a pure vapor in Ref. 28.

Let us consider the generalized boundary condition for the vapor, which is written in the form of a linear combination of the complete condensation and the diffuse reflection condition. To be more specific, let the velocity distribution $\Phi^{A}$ of outgoing molecules be given by the RHS of Eq. (4a) multiplied by $\alpha_{c}$ plus the RHS of Eq. (4b) with the superscript $B$ being replaced by $A$ multiplied by $1-\alpha_{c}$, where $\alpha_{c}$ $\left(0 \leq \alpha_{c} \leq 1\right)$ is a constant corresponding to the condensation coefficient. Then, the resulting condition is written as follows: at $r=1$, for $0 \leq \theta_{\zeta}<\pi / 2$,

$$
\begin{aligned}
& \Phi^{A}=\alpha_{c} \Delta P+\left(1-\alpha_{c}\right) \widetilde{\sigma}_{w}+\left(\zeta^{2}-\frac{5}{2}\right) \Delta T, \\
& \tilde{\sigma}_{w}=\frac{1}{2} \Delta T-2 \pi^{1 / 2}\left\langle\zeta \cos \theta_{\zeta} \Phi^{A} E^{A}\right\rangle_{w} .
\end{aligned}
$$

As for the noncondensable gas, we impose the diffuse reflection condition (4b) again. The solution of the boundary-value problem [Eqs. (2), (31), (4b), and (7)] is denoted by $\Phi_{G}^{\alpha}$ in the following.

Condition (31a) is equivalent to the complete condensation condition (4a) if the constant $\Delta P$ is replaced by $\alpha_{c} \Delta P$ $+\left(1-\alpha_{c}\right) \widetilde{\sigma}_{w}$. Taking into account this fact, we seek the solution $\Phi_{G}^{\alpha}$ in the following form: 


$$
\Phi_{G}^{\alpha}=\Phi_{P}^{\alpha} \Delta \widetilde{P}+\Phi_{T}^{\alpha} \Delta T .
$$

It is immediately seen that the above solution satisfies Eq. (2) and condition (7) at infinity. In addition, one can easily verify that $\Phi_{G}^{B}$ always satisfies condition (4b) irrespective of value of $\Delta \widetilde{P}$. Then, we determine $\Delta \widetilde{P}$ by substituting Eq. (32) with $\alpha=A$ into Eq. (31) and solving for it,

$$
\Delta \widetilde{P}=\frac{\alpha_{c} \Delta P-2 \pi^{1 / 2}\left(1-\alpha_{c}\right) u_{r T}^{A}(r=1) \Delta T}{\alpha_{c}+2 \pi^{1 / 2}\left(1-\alpha_{c}\right) u_{r P}^{A}(r=1)} .
$$

With the above $\Delta \widetilde{P}, \Phi_{G}^{A}$ satisfies condition (31). Therefore, the solution $\Phi_{G}^{\alpha}$ under the generalized boundary condition (31) can be constructed from the solution $\Phi_{P}^{\alpha}$ and $\Phi_{T}^{\alpha}$ under the complete condensation condition obtained in the present work. Note that $\Phi_{G}^{\alpha}$ for $\alpha_{c}=0$ corresponds to the solution for the heat transfer problem discussed in the last paragraph in Sec. IV A.

\section{CONCLUSIONS}

The vapor flow caused by evaporation or condensation on a spherical droplet has been investigated on the basis of kinetic theory in the case where another noncondensable gas is also present in the gas phase. Under the assumption of weak evaporation or condensation, the linearized model Boltzmann equation for gas mixtures (a linearized version of the model proposed by Hamel ${ }^{53}$ and that by Garzó et al. ${ }^{54}$ ) was solved with the complete condensation condition for the vapor and the diffuse reflection condition for the noncondensable gas being imposed on the surface of the droplet. The accurate numerical analysis by means of a finitedifference method was carried out, in which the hybrid scheme devised in Ref. 25 was employed to treat correctly the discontinuities of the velocity distribution function. The numerical results, together with the free-molecular solution and the asymptotic solution for small Knudsen numbers in Ref. 50, clearly exhibit the behavior of the mixture in the whole range of the Knudsen number.

In comparison with the case of a pure vapor studied in Ref. 28, it may be said that the main difference is seen in the behavior of the mass-flow rate $r^{2} u_{r P}^{A}$ for $(\Delta P, \Delta T)=(1,0)$ as a function of $k$. That is, $r^{2} u_{r P}^{A}$ vanishes as $k \rightarrow 0$ in the case of a mixture because of the obstruction caused by the noncondensable gas. We also made a comparison between the present results and Young's approximate formula, ${ }^{42}$ which gives an assessment of the performance of the latter formula. As a byproduct of the present analysis, the solution for the heat transfer problem between a solid sphere and a binary gas mixture was also obtained. Finally, we confirmed that a solution under the generalized boundary condition can be constructed from the present results.

\section{ACKNOWLEDGMENTS}

S.K. and K.A. thank Professor Shigeru Takata for his valuable remarks on the Onsager reciprocity relations.

\section{APPENDIX A: ASYMPTOTIC SOLUTION FOR LARGE $r$}

The velocity distribution function $\Phi^{\alpha}$ in the far field from the droplet $(r \gg 1)$ may be expected to be proportional to inverse power of $r$, i.e., $\Phi^{\alpha} \sim O\left(r^{-n}\right)$ (see Sec. III B and the third paragraph in Sec. III C). Consequently, $\partial \Phi^{\alpha} / \partial r$ $\sim \Phi^{\alpha} / r$ holds there, and thus the length scale of variation in $\Phi^{\alpha}$ is of the order of $L r$. In the far field $r>r_{0}\left(r_{0} \gg 1\right)$, therefore, the effective Knudsen number may be written as $\ell_{0}^{A} /\left(L r_{0}\right)=\mathrm{Kn} / r_{0}$, which will be smaller for larger $r_{0}$. With this fact in mind, we can derive the asymptotic solution $\Phi_{\text {far }}^{\alpha}$ for large $r$ from that for small $k$ given in Ref. 50. The result is

$$
\begin{aligned}
\Phi_{\text {far }}^{A}= & \frac{1}{k \Lambda} a r^{-1}+2 a r^{-2}\left[\zeta \cos \theta_{\zeta}+k r^{-1} \zeta^{2}\left(3 \cos ^{2} \theta_{\zeta}-1\right)\right] \\
& +b r^{-1}\left(\zeta^{2}-\frac{5}{2}\right)\left[1+k r^{-1} \zeta \cos \theta_{\zeta}\right. \\
& \left.+k^{2} r^{-2} \zeta^{2}\left(3 \cos ^{2} \theta_{\zeta}-1\right)\right], \\
\Phi_{\text {far }}^{B}= & -\frac{1}{k \Lambda \hat{n}_{0}^{B}} a r^{-1}+b r^{-1}\left(\hat{m}^{B} \zeta^{2}-\frac{5}{2}\right) \\
& \times\left[1+\Gamma k r^{-1} \zeta \cos \theta_{\zeta}+\Gamma^{2} k^{2} r^{-2} \zeta^{2}\left(3 \cos ^{2} \theta_{\zeta}-1\right)\right],
\end{aligned}
$$

where $\Lambda$ and $\Gamma$ are defined in Eq. (24) and $k$ is arbitrary. In the derivation of Eq. (A1), the following asymptotic behavior of the macroscopic quantities for $r \gg 1$, which also follows from the analysis for small $k$ in Ref. 50, is taken into account:

$$
u_{r}^{A}=a r^{-2}, \quad \tau^{A}=\tau^{B}=b r^{-1},
$$

where $a$ and $b$ are undetermined constants.

As described in Sec. III C, the above solution was used in the numerical analysis as the boundary condition at $r=r_{D}$ with sufficiently large $r_{D}$. In the computation, the constants $a$ and $b$ were determined numerically at each iteration step from the behavior of $u_{r}^{A}, \tau^{A}$, and $\tau^{B}$ in the far field.

\section{APPENDIX B: YOUNG'S APPROXIMATE FORMULA}

Young's approximate formula ${ }^{42}$ for the mass- and heatflow rates from the spherical droplet in the presence of a noncondensable gas may be written in terms of the present notation as follows:

$$
\begin{aligned}
& r^{2} u_{r P}^{*}=\frac{A_{q q} B_{m}}{2 \pi^{1 / 2} H}, \quad r^{2} u_{r T}^{*}=-\frac{A_{m q} B_{q}}{2 \pi^{1 / 2} H}, \\
& r^{2} Q_{r P}^{*}=-\frac{A_{q m} B_{m}}{2 \pi^{1 / 2} H}, \quad r^{2} Q_{r T}^{*}=\frac{A_{m m} B_{q}}{2 \pi^{1 / 2} H},
\end{aligned}
$$

with

$$
A_{m m}=F_{2} A_{2} \hat{n}_{0}^{B}+F_{1} A_{1} \mathrm{Kn}_{\mathrm{Y}}+\frac{A_{1} A_{2} G_{1} \mathrm{Kn}_{\mathrm{Y}}}{4 B_{2}},
$$




$$
\begin{aligned}
& A_{m q}=\frac{A_{1} A_{2} G_{1} \mathrm{Kn}_{\mathrm{Y}}}{2 B_{2}}, \quad A_{q m}=\frac{G_{1} B_{1} \mathrm{Kn}_{\mathrm{Y}}}{2}, \\
& A_{q q}=G_{2}+G_{1} B_{1} \mathrm{Kn}_{\mathrm{Y}}, \\
& H=A_{m m} A_{q q}-A_{m q} A_{q m}, \\
& B_{m}=A_{1} A_{2} \mathrm{Kn}_{\mathrm{Y}}, \quad B_{q}=B_{1} B_{2} \mathrm{Kn}_{\mathrm{Y}},
\end{aligned}
$$

and

$$
\begin{aligned}
& A_{1}=\frac{4}{\operatorname{Sc}}\left(\frac{R}{\kappa / m^{A}}\right)^{1 / 2}, \quad A_{2}=\frac{1}{1+\hat{n}_{0}^{B}}, \\
& B_{1}=\frac{8}{\operatorname{Pr}} \frac{\gamma}{\gamma+1}, \quad B_{2}=\frac{\gamma+1}{2(\gamma-1)}\left(\frac{R}{\kappa / m^{A}}\right)^{1 / 2}, \\
& F_{1}=\frac{1}{\alpha_{c}}-\frac{1}{2} F_{2}^{2}, \quad F_{2}=\frac{1}{1+2 \beta_{m} \mathrm{Kn}_{\mathrm{Y}}}, \\
& G_{1}=1-\frac{1}{2} G_{2}^{2}, \quad G_{2}=\frac{1}{1+2 \beta_{q} \mathrm{Kn}_{\mathrm{Y}}} .
\end{aligned}
$$

Here Sc is the Schmidt number, Pr is the Prandtl number, and $\mathrm{Kn}_{\mathrm{Y}}$ is the Knudsen number defined in Ref. 42. They are written in terms of the transport coefficients (e.g., Ref. 64), i.e., the viscosity $\mu$ of the mixture, thermal conductivity $\lambda$ of the mixture, and binary diffusion coefficient $D$ as

$$
\begin{aligned}
& \mathrm{Sc}=\frac{\mu}{\rho_{0} D}, \quad \operatorname{Pr}=\frac{\gamma R \mu}{(\gamma-1) \lambda}, \\
& \mathrm{Kn}_{\mathrm{Y}}=\frac{\mu\left(2 \pi R T_{0}\right)^{1 / 2}}{4 p_{0} L},
\end{aligned}
$$

with $\rho_{0}=m^{A} n_{0}^{A}+m^{B} n_{0}^{B}$. The $R$ is the specific gas constant of the mixture per unit mass and $\gamma$ is a constant defined by the following equation:

$$
\frac{\gamma+1}{\gamma-1}\left(1+\hat{n}_{0}^{B}\right)=\frac{\gamma^{A}+1}{\gamma^{A}-1}\left(\frac{\kappa / m^{A}}{R}\right)^{1 / 2}+\frac{\gamma^{B}+1}{\gamma^{B}-1} \hat{n}_{0}^{B}\left(\frac{\kappa / m^{B}}{R}\right)^{1 / 2},
$$

with $\gamma^{A}$ and $\gamma^{B}$ being the specific heat ratios of the vapor and noncondensable gas. The $\alpha_{c}$ appearing in Eq. (B3c) is the condensation (or evaporation) coefficient, and $\beta_{m}$ and $\beta_{q}$ in Eqs. (B3c) and (B3d) are positive constants defining the Knudsen-continuum interface in the Langmuir model. Note that Onsager's reciprocity relation (18) and inequality (19) hold in formula (B1).

Formula (B1) is compared to the present results in Sec. IV A (see Fig. 4 and also Figs. 1 and 2 in the supplementary material $^{62}$ ). In the comparison, the specific gas constant $R$ of the mixture appearing in the above equations is defined as $R=\left(n_{0} / \rho_{0}\right) \kappa$, the specific heat ratios are set as $\gamma^{A}=\gamma^{B}=5 / 3$ (i.e., the value for a monoatomic ideal gas), and $\alpha_{c}=1$. As for the transport coefficients, the following expressions for the model equation are used (see Refs. 53 and 54):

$$
\begin{aligned}
\mu & =\frac{p_{0}^{A}}{K^{A A} n_{0}^{A}+K^{A B} n_{0}^{B}}+\frac{p_{0}^{B}}{K^{A B} n_{0}^{A}+K^{B B} n_{0}^{B}}, \\
\lambda & =\frac{5}{2} \kappa\left(\frac{p_{0}^{A} / m^{A}}{K^{A A} n_{0}^{A}+K^{A B} n_{0}^{B}}+\frac{p_{0}^{B} / m^{B}}{K^{A B} n_{0}^{A}+K^{B B} n_{0}^{B}}\right), \\
D & =\frac{\left(m^{A}+m^{B}\right) \kappa T_{0}}{m^{A} m^{B} K^{A B} n_{0}} .
\end{aligned}
$$

Using the above expression for $\mu$, we can derive the relation between $\mathrm{Kn}_{\mathrm{Y}}$ and $k$ as

$$
\mathrm{Kn}_{\mathrm{Y}}=\frac{\pi^{1 / 2}}{4} \frac{1+\Gamma \hat{n}_{0}^{B}}{\left(1+\hat{n}_{0}^{B}\right)^{1 / 2}\left(1+\hat{m}^{B} \hat{n}_{0}^{B}\right)^{1 / 2}} k,
$$

with $\Gamma$ being defined in Eq. (24). The parameters $\beta_{m}$ and $\beta_{q}$ are set as $\left(\beta_{m}, \beta_{q}\right)=(1.5,0.75)$ so that formula (B1) best approximates the maximum values of $r^{2} u_{r P}^{A}$ for $\hat{n}_{0}^{B}=0.1$ in Figs. 1(a), 2(a), and 3(a).

${ }^{1}$ T. Ytrehus, "Molecular-flow effects in evaporation and condensation at interfaces," Multiphase Sci. Technol. 9, 205 (1997).

${ }^{2}$ C. Cercignani, Rarefied Gas Dynamics: From Basic Concepts to Actual Calculations (Cambridge University Press, Cambridge, 2000).

${ }^{3}$ A. K. Rebrov, "Nonequilibrium processes at evaporating and condensing surfaces," in Rarefied Gas Dynamics, edited by T. J. Bartel and M. A. Gallis (AIP, Melville, NY, 2001), p. 557.

${ }^{4}$ V. I. Roldughin and V. M. Zhdanov, "Non-equilibrium thermodynamics and kinetic theory of gas mixtures in the presence of interfaces," Adv. Colloid Interface Sci. 98, 121 (2002).

${ }^{5}$ Y. Sone, Kinetic Theory and Fluid Dynamics (Birkhäuser, Boston, 2002).

${ }^{6}$ Y. Sone, Molecular Gas Dynamics, Theory, Techniques, and Applications (Birkhäuser, Boston, 2007).

${ }^{7}$ M. N. Kogan and N. K. Makashev, "Role of the Knudsen layer in the theory of heterogeneous reactions and in flows with surface reactions," Fluid Dyn. 6, 913 (1971).

${ }^{8}$ Y. Sone and Y. Onishi, "Kinetic theory of evaporation and condensation," J. Phys. Soc. Jpn. 35, 1773 (1973).

${ }^{9}$ C. E. Siewert and J. R. Thomas, Jr., "Half-space problems in the kinetic theory of gases," Phys. Fluids 16, 1557 (1973).

${ }^{10}$ J. W. Cipolla, Jr., H. Lang, and S. K. Loyalka, "Kinetic theory of condensation and evaporation. II," J. Chem. Phys. 61, 69 (1974).

${ }^{11} \mathrm{~T}$. Ytrehus, "Theory and experiments on gas kinetics in evaporation," in Rarefied Gas Dynamics, edited by J. L. Potter (AIAA, New York, 1977), Pt. II, p. 1197.

${ }^{12}$ A. A. Abramov and M. N. Kogan, "Conditions for supersonic condensation of gas," Sov. Phys. Dokl. 29, 763 (1984).

${ }^{13}$ A. P. Kryukov, "One-dimensional steady condensation of vapor velocities comparable to the velocity of sound," Fluid Dyn. 20, 487 (1985).

${ }^{14}$ Y. Sone, K. Aoki, and I. Yamashita, "A study of unsteady strong condensation on a plane condensed phase with special interest in formation of steady profile," in Rarefied Gas Dynamics, edited by V. Boffi and C. Cercignani (Teubner, Stuttgart, 1986), Vol. II, p. 323.

${ }^{15} \mathrm{Y}$. Sone and H. Sugimoto, "Strong evaporation from a plane condensed phase," in Adiabatic Waves in Liquid-Vapor Systems, edited by G. E. A. Meier and P. A. Thompson (Springer-Verlag, Berlin, 1990), p. 293.

${ }^{16}$ Y. P. Pao, "Application of kinetic theory to the problem of evaporation and condensation," Phys. Fluids 14, 306 (1971).

${ }^{17}$ J. R. Thomas, Jr., T. S. Chang, and C. E. Siewert, "Reverse temperature gradient in the kinetic theory of evaporation," Phys. Rev. Lett. 33, 680 (1974).

${ }^{18}$ Y. Sone, T. Ohwada, and K. Aoki, "Evaporation and condensation of a rarefied gas between its two parallel plane condensed phases with different temperatures and negative temperature-gradient phenomenon: Numerical analysis of the Boltzmann equation for hard-sphere molecules," in Mathematical Aspects of Fluid and Plasma Dynamics, Lecture Notes in Mathematics Vol. 1460, edited by G. Toscani, V. Boffi, and S. Rionero (Springer-Verlag, Berlin, 1991), p. 186. 
${ }^{19}$ R. E. Sampson and G. S. Springer, "Condensation on and evaporation from droplets by a moment method," J. Fluid Mech. 36, 577 (1969); 40, 859(E) (1970).

${ }^{20}$ C. J. Knight, "Evaporation from a cylindrical surface into vacuum," J. Fluid Mech. 75, 469 (1976).

${ }^{21}$ Y. Sone and Y. Onishi, "Kinetic theory of evaporation and condensation: Hydrodynamic equation and slip boundary condition," J. Phys. Soc. Jpn. 44, 1981 (1978)

${ }^{22}$ V. G. Chernyak and A. Ye. Margilevskiy, "The kinetic theory of heat and mass transfer from a spherical particle in a rarefied gas," Int. J. Heat Mass Transfer 32, 2127 (1989).

${ }^{23}$ D. Sibold and H. M. Urbassek, "Kinetic study of evaporation flows from cylindrical jets," Phys. Fluids A 3, 870 (1991).

${ }^{24} \mathrm{~J}$. B. Young, "The condensation and evaporation of liquid droplets in a pure vapour at arbitrary Knudsen number," Int. J. Heat Mass Transfer 34, 1649 (1991).

${ }^{25} \mathrm{H}$. Sugimoto and Y. Sone, "Numerical analysis of steady flows of a gas evaporating from its cylindrical condensed phase on the basis of kinetic theory," Phys. Fluids A 4, 419 (1992).

${ }^{26}$ Y. Sone and H. Sugimoto, "Kinetic theory analysis of steady evaporating flows from a spherical condensed phase into a vacuum," Phys. Fluids A 5, 1491 (1993); 7, 2096(E) (1995).

${ }^{27}$ Y. Sone, S. Takata, and M. Wakabayashi, "Numerical analysis of a rarefied gas flow past a volatile particle using the Boltzmann equation for hardsphere molecules," Phys. Fluids 6, 1914 (1994).

${ }^{28}$ S. Takata, Y. Sone, D. Lhuillier, and M. Wakabayashi, "Evaporation from or condensation onto a sphere: Numerical analysis of the Boltzmann equation for hard-sphere molecules," Comput. Math. Appl. 35, 193 (1998).

${ }^{29}$ Y. Sone, K. Aoki, and T. Doi, "Kinetic theory analysis of gas flows condensing on a plane condensed phase: Case of a mixture of a vapor and a noncondensable gas," Transp. Theory Stat. Phys. 21, 297 (1992).

${ }^{30} \mathrm{~K}$. Aoki, S. Takata, and S. Kosuge, "Vapor flows caused by evaporation and condensation on two parallel plane surfaces: Effect of the presence of a noncondensable gas," Phys. Fluids 10, 1519 (1998).

${ }^{31} \mathrm{~S}$. Taguchi, K. Aoki, and S. Takata, "Vapor flows condensing at incidence onto a plane condensed phase in the presence of a noncondensable gas. I. Subsonic condensation," Phys. Fluids 15, 689 (2003).

${ }^{32}$ K. Aoki, M. Hatano, S. Kosuge, and S. Takata, "Diffusiophoresis of a spherical volatile particle," in Rarefied Gas Dynamics, edited by M. Capitelli (AIP, Melville, NY, 2005), p. 713.

${ }^{33}$ A. Frezzotti, "Kinetic theory description of the evaporation of multicomponent substances," in Rarefied Gas Dynamics, edited by C. Shen (Peking University Press, Beijing, 1997), p. 837.

${ }^{34} \mathrm{~S}$. Takata, "Kinetic theory analysis of the two-surface problem of a vaporvapor mixture in the continuum limit," Phys. Fluids 16, 2182 (2004).

${ }^{35} \mathrm{~S}$. Takata and F. Golse, "Half-space problem of the nonlinear Boltzmann equation for weak evaporation and condensation of a binary mixture of vapors," Eur. J. Mech. B/Fluids 26, 105 (2007).

${ }^{36}$ S. N. Pandis, A. S. Wexler, and J. H. Seinfeld, "Dynamics of tropospheric aerosols," J. Phys. Chem. 99, 9646 (1995).

${ }^{37}$ A. Colaprete and O. B. Toon, "Carbon dioxide clouds in an early dense Martian atmosphere," J. Geophys. Res., [Planets] 108, 5025, doi:10.1029/ 2002JE001967 (2003).

${ }^{38}$ S. S. Sazhin, "Advanced models of fuel droplet heating and evaporation," Prog. Energy Combust. Sci. 32, 162 (2006).

${ }^{39}$ S. S. Sazhin, I. N. Shishkova, A. P. Kryukov, V. Yu. Levashov, and M. R. Heikal, "Evaporation of droplets into a background gas: Kinetic modelling," Int. J. Heat Mass Transfer 50, 2675 (2007).

${ }^{40}$ X. S. Luo, B. Prast, M. E. H. Van Dongen, H. W. M. Hoeijmakers, and J. M. Yang, "On phase transition in compressible flows: Modelling and validation," J. Fluid Mech. 548, 403 (2006).
${ }^{41}$ N. A. Fuchs and A. G. Sutugin, Highly Dispersed Aerosols (Ann Arbor Science, Ann Arbor, 1970).

${ }^{42}$ J. B. Young, "The condensation and evaporation of liquid droplets at arbitrary Knudsen number in the presence of an inert gas," Int. J. Heat Mass Transfer 36, 2941 (1993).

${ }^{43}$ P. N. Shankar, "A kinetic theory of steady condensation," J. Fluid Mech. 40, 385 (1970).

${ }^{44}$ I. Ivchenko, "Evaporation (condensation) theory of spherical particles with all Knudsen numbers," J. Colloid Interface Sci. 120, 1 (1987).

${ }^{45} \mathrm{M}$. Sitarski and B. Nowakowski, "Condensation rate of trace vapor on Knudsen aerosols from the solution of the Boltzmann equation," J. Colloid Interface Sci. 72, 113 (1979).

${ }^{46} \mathrm{X}$. Qu and E. J. Davis, "Droplet evaporation and condensation in the near-continuum regime," J. Aerosol Sci. 32, 861 (2001).

${ }^{47}$ S. K. Loyalka, "Condensation on a spherical droplet," J. Chem. Phys. 58, 354 (1973)

${ }^{48}$ S. K. Loyalka, S. A. Hamoodi, and R. V. Tompson, "Isothermal condensation on a spherical particle," Phys. Fluids A 1, 358 (1989).

${ }^{49} \mathrm{~V}$. Chernyak, "The kinetic theory of droplet evaporation," J. Aerosol Sci. 26, 873 (1995).

${ }^{50}$ Y. Onishi, "The spherical-droplet problem of evaporation and condensation in a vapour-gas mixture," J. Fluid Mech. 163, 171 (1986).

${ }^{51} \mathrm{~S}$. Takeuchi and Y. Onishi, "Weakly nonlinear analysis for evaporation and condensation of a vapor-gas mixture from a spherical condensed phase," in Rarefied Gas Dynamics, edited by C. Shen (Peking University Press, Beijing, 1997), p. 877.

${ }^{52}$ Y. Sone and S. Takata, "Discontinuity of the velocity distribution function in a rarefied gas around a convex body and the $\mathrm{S}$ layer at the bottom of the Knudsen layer," Transp. Theory Stat. Phys. 21, 501 (1992).

${ }^{53}$ B. B. Hamel, "Kinetic model for binary gas mixtures," Phys. Fluids 8 , 418 (1965)

${ }^{54}$ V. Garzó, A. Santos, and J. J. Brey, "A kinetic model for a multicomponent gas," Phys. Fluids A 1, 380 (1989).

${ }^{55}$ Y. Sone and K. Aoki, "A similarity solution of the linearized Boltzmann equation with application to thermophoresis of a spherical particle," J. Mec. Theor. Appl. 2, 3 (1983).

${ }^{56}$ F. Sharipov, "Onsager-Casimir reciprocity relations for open gaseous systems at arbitrary rarefaction I. General theory for single gas," Physica A 203, 437 (1994).

${ }^{57} \mathrm{~F}$. Sharipov, "Onsager-Casimir reciprocity relations for open gaseous systems at arbitrary rarefaction III. Theory and its application for gaseous mixtures," Physica A 209, 457 (1994).

${ }^{58}$ S. Takata, "Symmetry of the linearized Boltzmann equation and its application," J. Stat. Phys. 136, 751 (2009).

${ }^{59} \mathrm{~S}$. Takata, "Symmetry of the linearized Boltzmann equation and its application II. Entropy production and Onsager-Casimir relation," J. Stat. Phys. 136, 945 (2009).

${ }^{60}$ Y. Sone, "Asymptotic theory of flow of rarefied gas over a smooth boundary I," in Rarefied Gas Dynamics, edited by L. Trilling and H. Y. Wachman (Academic, New York, 1969), Vol. I, p. 243.

${ }^{61}$ S. Takata and Y. Sone, "Flow induced around a sphere with a non-uniform surface temperature in a rarefied gas, with application to the drag and thermal force problems of a spherical particle with an arbitrary thermal conductivity,” Eur. J. Mech. B/Fluids 14, 487 (1995).

${ }^{62}$ See supplementary material at http://dx.doi.org/10.1063/1.3432130 for tables of numerical data and supplementary figures.

${ }^{63}$ F. Peters and B. Paikert, "Measurement and interpretation of growth and evaporation of monodispersed droplets in a shock tube," Int. J. Heat Mass Transfer 37, 293 (1994).

${ }^{64}$ S. Chapman and T. G. Cowling, The Mathematical Theory of NonUniform Gases, 3rd ed. (Cambridge University Press, Cambridge, 1970). 